\title{
1 Design of novel CV-N variants: Modulation of binding dynamics through distal mutations
}

3 I. Can Kazan ${ }^{1,2} \uparrow$, Prerna Sharma ${ }^{1}$, Andrey Bobkov ${ }^{3}$, Raimund Fromme ${ }^{1}$, Giovanna Ghirlanda ${ }^{1 *}$,

4 S. Banu Ozkan ${ }^{2 *}$

$6 \quad{ }^{1}$ School of Molecular Sciences, Arizona State University, Tempe, AZ

$7 \quad{ }^{2}$ Center for Biological Physics and Department of Physics, Arizona State University, Tempe, AZ

$8 \quad{ }^{3}$ Sanford Burnham Prebys Medical Discovery Institute, La Jolla, CA

$10 *$ Corresponding authors

11 Email: Giovanna.Ghirlanda@asu.edu

14 These authors contributed equally to this work.

16 Abstract

We develop a computational approach to identify distal residues that allosterically

19 modulate dynamics of binding sites by combining dynamic coupling with statistical analysis of

20 co-evolution. Putative mutants of the predicted allosteric sites are subjected to Adaptive BP-Dock

21 for binding analysis. Here, we apply this method to a small lectin, Cyanovirin-N (CV-N), that

22 selectively binds to dimannose. Our method points out mutations on I34, that is $16 \AA$ away from

23 binding pocket, can modulate binding. Experimental characterization confirms that I34K abolishes 
24 binding, while I34Y increases affinity. Increased affinity is not due to changes in the binding

25 region, which are conserved in the crystal structure. However, ITC analysis reveals an opposite

26 entropic contribution, suggesting that modulation of dynamics is responsible for the change in

27 binding affinity. Our results point to a novel approach to identify and substitute distal sites by

28 integrating evolutionary inference with protein dynamics in glycan-binding proteins to improve

29 binding affinity.

\section{Introduction}

Naturally occurring proteins exhibit a fine balance between protein stability and biological

33 function. Protein sequence underpins any possible protein function, yet deciphering this encoded

34 information is still a challenge (Salinas and Ranganathan, 2018). Evolutionarily related proteins

35 display co-variation at specific sites, pointing to the importance of interactions among these

36 residues in governing 3-D structure and function. Sequence-based prediction methods involving

37 multiple sequence alignment (MSA) to map co-evolved positions through information theory

38 based approaches usually identify the residue pairs that have direct interactions (i.e. in contact in

39 3-D structure), yet mask allosteric coupling between residues far from each other (long range

40 interactions) or phylogenetic effects (Anishchenko et al., 2017; Jones et al., 2012; Kamisetty et al.,

41 2013). As a result, it is hard to identify important sites distal from functional sites (binding or

42 active sites that modulate function). However, directed evolution studies have shown that these

43 long range interactions between distal residues and binding/active site play a role in function, and

44 substitutions on these distal sites are crucial to optimize leads in computational protein design

45 (Acar et al., 2020; Barrientos et al., 2006; Campbell et al., 2016, 2018; Campitelli et al., 2020a; 
46 Keeffe et al., 2011; Otten et al., 2018; Risso et al., 2018; Romero and Arnold, 2009; Tokuriki et

47 al., 2012; Yuan et al., 2005; Zhang et al., 2006).

To overcome this challenge in protein design, here we combine co-evolution analyses binding affinity by altering the conformational dynamics of the binding pocket. Thus, after

54 identifying an allosteric site, we first computationally screened how mutations modulate binding affinity using our in-house flexible docking method Adaptive BP-dock and then validated our predictions experimentally (Bolia and Ozkan, 2016).

The model protein chosen for characterizing allosteric modulation of binding mediated by

natural lectin isolated from cyanobacterium Nostoc ellipsosporum and has been studied extensively as a model to investigate protein interactions with glycans (Barrientos et al., 2003; the CV-N homology family contain two sequential repeats of about 50 residues. While residues in

64 the core region are highly conserved, residues involved in dimannose binding are not highly 65 conserved resulting in differences in specificity and selectivity of the binding pockets (Koharudin 66 et al., 2008; Koharudin and Gronenborn, 2013; Patsalo et al., 2011; Percudani et al., 2005; Qi et 67 al., 2009). Most homologs characterized so far show high conservation of the structural binding 68 units, but they lose the specificity for dimannose that is a hallmark of CV-N recognition, and bind 
69 to oligomannose and other glycans at much higher micromolar concentrations (Koharudin et al.,

70 2009, 2008; Matei et al., 2016; Woodrum et al., 2013).

74 linker. Despite almost having identical structures, the domains show relatively low sequence

75 homology (28\% sequence identity, and 52\% similarity). Functionally, they both bind dimannose,

76 yet the affinity is quite different, with domain B having tighter binding affinity $\left(\mathrm{K}_{\mathrm{d}}=15.3 \mu \mathrm{M}\right)$,

77 and domain A showing weak affinity $\left(\mathrm{K}_{\mathrm{d}}=400 \mu \mathrm{M}\right)($ Balzarini, 2007; Bolmstedt et al., 2001; Li

78 et al., 2015). To simplify our analysis we used a designed CV-N variant, P51G-m4, that contains

79 a single high-affinity dimannose-binding site (domain B), folds exclusively as a monomer in

80 physiological conditions, and is more stable to thermal denaturation than wild type (Fromme et

81 al., 2008, 2007).

Application of our approach on P51G-m4 identified position I34, which is $16 \AA$ away from

84 the binding pocket, as highly coupled and co-evolved with the binding residues of domain B. Based

85 on the co-variation with binding pocket residues, three variants of I34 (K, L, Y) were selected for

86 further analyses. The mutants were modeled by GROMACS Molecular Dynamics (MD)

87 simulations and subjected to docking using Adaptive BP dock. The docking results uncovered that

88 each variant acts differently to binding of dimannose. Experimental biophysical characterization

89 of the mutants confirmed that (i) I34Y increases the binding affinity, (ii) I34L is neutral and

90 consistent with the template, and (iii) I34K abolishes binding affinity towards dimannose. The

91 crystal structure of I34Y with and without dimannose shows high conservation of the overall fold 
92 and particularly its binding motif compared to P51G-m4, despite the enhancement in binding

93 affinity. Conformational dynamics analysis by MD revealed that substitution on I34 modulates

94 equilibrium distribution of the conformations of the binding pocket towards open pocket. In

95 addition, dynamic flexibility index (DFI) analysis shows that I34Y mutant results in rigidification

96 of binding residues T57, E41 and N42, in turns decreasing the entropy cost of binding and leading

97 to the favorable binding affinity. A favorable entropy term in binding by I34Y variant is supported

98 by ITC studies. Our findings indicate that it is possible to modulate ligand binding through

99 mutation at a distal location, based on long distance dynamic coupling with binding sites, and

100 present a novel computational approach to identify those sites.

101

102 Results and Discussion

103

104 Combining long-range dynamic coupling analysis with co-evolution allows to identify distal

105 sites that contribute to binding affinity.

The binding pocket of domain $\mathrm{B}$ of $\mathrm{CV}-\mathrm{N}$ has been subjected to intense scrutiny to glean

108 the origin of its binding specificity for dimannose (Bewley, 2001; Bolia et al., 2014b; Botos and

109 Wlodawer, 2003; Li et al., 2015; Vorontsov and Miyashita, 2009). Previous mutational studies on

110 the binding pocket residues have shown their importance in modulating interaction with

111 dimannose along with a role in modulating water-mediated interactions (Barrientos et al., 2006;

112 Bolia et al., 2014b; Chang and Bewley, 2002; Matei et al., 2008). So far all substitutions of the

113 binding residues led to decreased binding affinity for dimannose on domain B (Bolia et al., 2014b;

114 Fujimoto and Green, 2012; Kelley et al., 2002; Matei et al., 2011; Ramadugu et al., 2014). 
115 Furthermore, evolutionary analyses reassures the majority of the binding site residues are

116 conserved in CV-N glycan interactions, suggesting that it is harder to enhance affinity through

117 mutations of these conserved binding sites. (Koharudin et al., 2008; Percudani et al., 2005). Here,

118 we take a completely different approach and aim to identify positions that are not directly

119 interacting with glycans but can potentially impact glycan binding through modulating the protein

120 ensemble in equilibrium. These positions could be far away yet can modulate the binding by

121 altering the conformational dynamics of the binding pocket due to long-distance communication

122 through fluctuations arising anisotropic interaction within the 3-D network of interactions (i.e.,

123 dynamic allosteric communications) (Acar et al., 2020; Campbell et al., 2016; Gunasekaran et al.,

124 2004; Lu and Liang, 2009; Wodak et al., 2019). We call positions that exhibit dynamic coupling

125 with the binding sites and mediate long-distance communication dynamic allosteric residue

126 coupling (DARC) spots. Our protein dynamics analyses on different protein families, particularly

127 on ancestral resurrected proteins have revealed that many proteins harbor DARC spots and that

128 substitutions on the DARC spots play a critical role in modulating the function for adaptation to

129 new environments (Kumar et al., 2015b; Modi and Ozkan, 2018; Ose et al., 2020).

To seek DARC spots in the CV-N scaffold we first applied our Dynamic Flexibility Index

132 (DFI) and Dynamic coupling Index (DCI) analyses. DFI score measures the resilience of a position

133 by computing the total fluctuation response of a position when each position in the protein is

134 perturbed with a random unit force and is related to the flexibility/rigidity of a given position (see

135 methods). Our earlier work has shown that rigid positions, which we call hinge sites, contribute

136 the most to the equilibrium dynamics that sample conformations in native state and distal hinge 
137 positions that are coupled to the active site could modulate dynamics to alter function (Gerek and

138 Ozkan, 2011; Kumar et al., 2015b; Larrimore et al., 2017; Modi and Ozkan, 2018).

As Natural selection strongly shapes the mutational landscape through protein dynamics

141 (Campitelli et al., 2020a; Modi et al., 2021), we combine evolutionary inference obtained from

142 multiple sequence alignment with position specific, dynamics-based DCI and DFI profiles of CVN

143 to modify the binding affinity of a lectin towards a specific glycan. Particularly, we searched for

144 distal rigid positions (exhibiting \%DFI values 0.2 or lower and at least $15 \AA$ away from binding

145 residues) that are dynamically coupled and co-evolved with the binding pocket (showing 0.7 or

146 higher dynamic coupling with the binding site) (Supplementary file 1 table 1).

149 implicating this distal location in transferring allosteric communication and controlling the 150 dynamics of the binding site, over a $16 \AA$ distance (Table S1). 


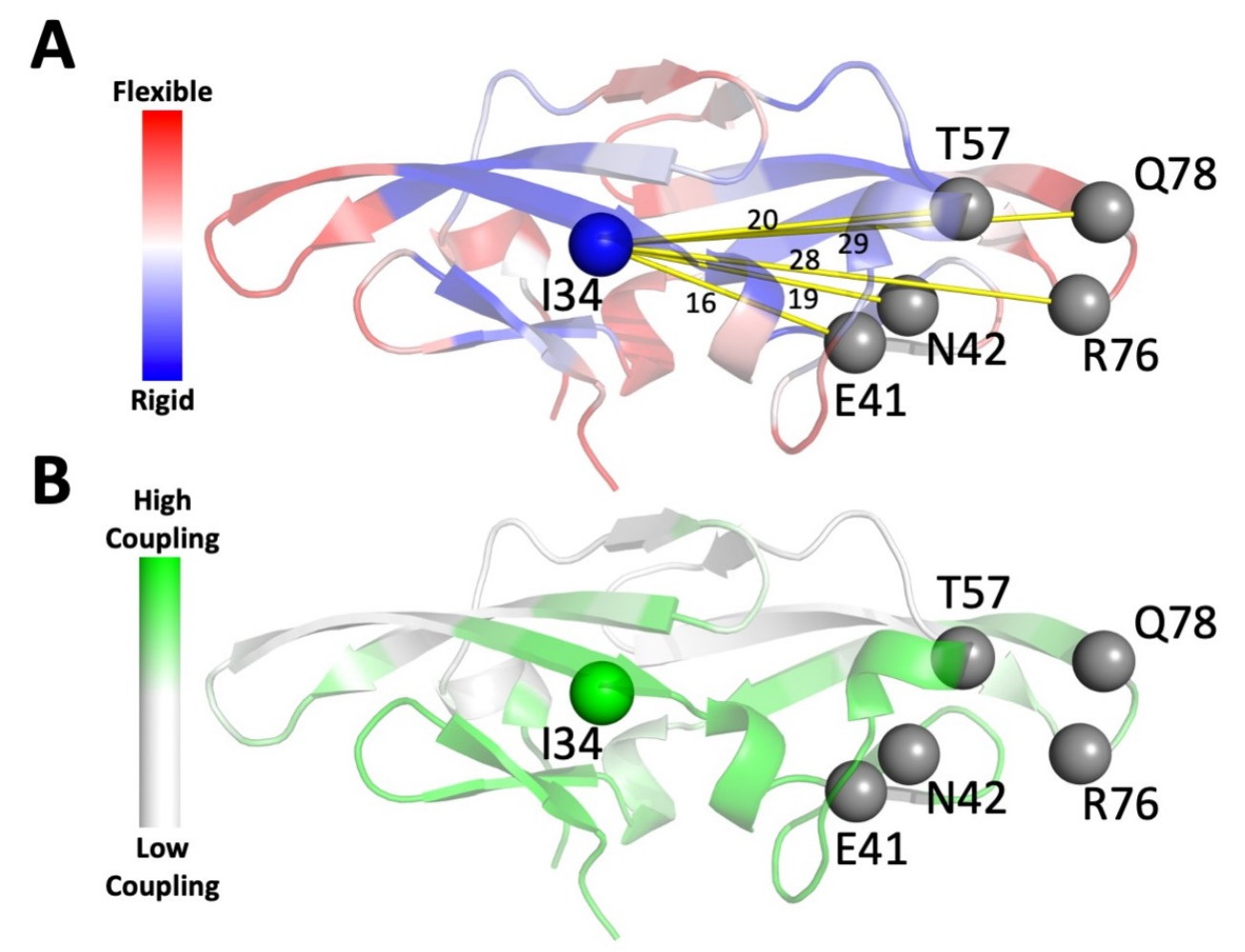

Fig 1 A) The DFI profile mapped onto CV-N structure within a color spectrum of red white blue where red presents positions with high DFI, (very flexibile sites), and blue presents positions with low DFI values (rigid sites). Residue I34 shows low DFI score, suggesting its critical role in mediating the collective motion and its importance in function. B) DCI profile projected on CV-N structure in a color-coded spectrum sites exhibiting high DCI are green. Residue I34 is found as highly dynamically coupled to binding site residues. The color-code maps from dynamics metrics suggest I34 can potentially be an allosteric modulator for binding where substitutions can alter glycan interactions.

To further analyze potential impact of position I34 on binding, we applied the DCI metric

154 to capture its long-distance dynamic coupling with the binding residues. DCI metric shows the 155 fluctuation response of a position of interest when a functional group (i.e., binding sites) is 
156 perturbed by unit forces relative to its fluctuation response to a perturbation to any other positions

157 in the protein. In other words, it measures how much a residue of interest is dynamically coupled

158 to another position, particularly functionally important positions (Campitelli et al., 2018;

159 Larrimore et al., 2017). We found that residue I34 is highly coupled to binding site residues of

160 domain B of CV-N (Fig 1B) (Table S1) (Supplementary file 1 table 1), suggesting that

161 substitutions at this position could modulate binding dynamics as observed in other systems

162 (Campitelli et al., 2020a; Risso et al., 2018). Additionally, we applied co-evolutionary analyses to

163 estimate evolutionary coupling between residue I34 and binding site residues (Supplementary file

1641 table 1) (Hopf et al., 2019; Ma et al., 2015; Simonetti et al., 2013) (See methods). We found that

165 I34 is co-evolved with five binding site positions (Table S1). When both conformational dynamics

166 analyses, and co-evolution analyses are used to select residue positions that fit our criteria, I34

167 emerges as the one and only hinge position that is more than $15 \AA$ away and the only co-evolved

168 position exhibiting dynamic coupling with the binding site. We note that it is remarkable

169 considering hundred locations in CV-N. only one residue that is distal and both exhibiting high

170 dynamic coupling and co-evolved with the binding sites. emerged from our analyses. Therefore,

171 we selected I34 for further investigation.

173 Substitutions at a distal site yield to change in binding affinity from enhancing to abolishing

174 glycan binding

175

Next, we selected type of amino acid substitutions on position I34 (i.e., I34 variants) based

177 on the amino-acid frequencies observed using MSA of CV-N homolog sequences. To this aim, we

178 first generated a multiple sequence alignment using CVN family members whose binding site 
residues have identical or similar amino-acid composition to P51G-m4 CV-N. We then obtained the frequency of amino acid types at position 34 for these proteins. This analysis indicates that the most frequently observed amino acids at this position are L and Y. As the earlier studies displays

182 (Jana et al., 2014; Modi et al., 2021; Morcos, 2020; Morcos et al., 2014, 2013), negative selection

183 (i.e. elimination of amino-acid types that are detrimental to the folding) is a major force in shaping

184 the mutational landscape. We can confidently assume that these frequently observed residue types

185 on positions 34 do not disrupt folding due to their presence in the MSA. We also selected K as a 186 mutant, because it is the only positively charged amino acid observed at position 187 34. (Supplementary file 1 table 2 ) to Molecular Dynamics simulations (Abraham et al., 2015; Spoel et al., 2005). At the end of the

191 simulations, the conformations are clustered by their root-mean-square deviation (RMSD), and the 192 most frequently sampled conformation is selected for docking analysis using our in house 193 algorithm Adaptive BP-Dock (Bolia and Ozkan, 2016), which incorporates both ligand and 194 receptor flexibility into computational methods to accurately sample binding induced 195 conformations during docking. These features are particularly important when docking highly 196 flexible ligands such as glycans, and we previously used Adaptive BP-Dock to analyze and predict 197 binding to dimannose in a series of CV-N binding site mutants (Bolia et al., 2014b; Li et al., 2015; 198 Woodrum et al., 2013). Here, we apply Adaptive BP-Dock to experimentally characterized wild199 type CV-N, and its variants: P51G-m4, mutDB (a mutant in which binding by domain B has been 200 obliterated) for benchmarking, and then compare their binding score energies to those of I34 201 variants. Adaptive BP-Dock has previously used to analyze different single point mutants of P51G- 
$202 \mathrm{~m} 4 \mathrm{CV}-\mathrm{N}$ and good correlations were obtained between X-scores binding energy scores and

203 experimentally measured binding affinities $\left(\mathrm{K}_{\mathrm{d}}\right)$ (Bolia et al., 2014b; Li et al., 2015). These

204 previous studies also showed that $-6 \mathrm{kcal} / \mathrm{mol} \mathrm{X}$-score binding energy is a good threshold to

205 differentiate dimannose binding CV-Ns from mutants that cannot bind.

209 affinity in a wide range: I34Y variant leads to an increase in binding affinity (beneficial), I34K

210 decreases the binding affinity (deleterious), and I34L yields no change (neutral). It is interesting

211 to observe that a distal site can modulate the binding with a wide range of outcome based on amino-

212 acid substitutions. This finding has also been observed for allosterically regulated enzymes such

213 as LacI where different amino-acid substitutions on non-conserved sites lead to gradual changes

214 in function, acting like a rheostatic switch to modulate function through conformational dynamics

215 (Campitelli et al., 2020b). 
Table 1. The predicted binding affinities of domain B and comparison with experimental ITC data for P51G-m4 and its I34 variants. Docking results shows I34Y mutant has higher binding towards dimannose improving on its P51G-m4 template binding affinity.

\begin{tabular}{|c|c|c|c|c|c|}
\hline Protein & $\begin{array}{c}\text { Predicted } \\
\text { Binding } \\
\text { Score } \\
\mathbf{k c a l} / \mathbf{m o l})\end{array}$ & $\begin{array}{c}\text { ITC } \\
\mathbf{d i m a n n o s e} \\
\mathbf{K}_{\mathbf{d}}(\boldsymbol{\mu M})\end{array}$ & $\begin{array}{c}\text { ITC } \\
\mathbf{d i m a n n o s e} \\
\Delta \mathbf{H}(\mathbf{k c a l} / \mathbf{m o l})\end{array}$ & $\begin{array}{c}\text { ITC } \\
\mathbf{d i m a n n o s e} \\
\mathbf{T} \mathbf{S}(\mathbf{k c a l} / \mathbf{m o l}) \\
\mathbf{( T = 2 9 8 K )}\end{array}$ & $\begin{array}{c}\text { ITC } \\
\mathbf{d i m a n n o s e}(\mathbf{k c a l} / \mathbf{m o l})\end{array}$ \\
\hline Wild Type & -7.08 & $16 \pm 1$ & $-12.5 \pm 0.3$ & $-6.0 \pm 0.1$ & \\
\hline mutDB & -5.97 & No-binding & & & \\
\hline P51G-m4 & -6.62 & $117 \pm 3$ & $-12.3 \pm 0.3$ & $-7.0 \pm 0.3$ & $-5.3 \pm 0.3$ \\
\hline P51G-m4-I34K & -5.85 & No-binding & No-binding & No-binding & No-binding \\
\hline P51G-m4-I34L & -6.19 & $148 \pm 2$ & $-9.6 \pm 0.1$ & $-4.4 \pm 0.1$ & $-5.2 \pm 0.1$ \\
\hline P51G-m4-I34Y & -6.75 & $64 \pm 5$ & $-4.35 \pm 0.1$ & $1.32 \pm 0.2$ & $-5.67 \pm 0.2$ \\
\hline
\end{tabular}

The computational predictions were validated by experimental characterization of the

225 mutant proteins, prepared as described in Methods.

We assessed the folding and thermal stability of the mutants by circular dichroism (CD) spectroscopy. The Far-UV CD spectra of the mutants showed that all the mutants are well folded

229 and adopt a fold similar to the parent protein, characterized by a single negative band centered at $230216 \mathrm{~nm}$. Thermal denaturation was used to measure apparent melting temperature $\left(\mathrm{T}_{\mathrm{m}}\right)$ values. We

231 found that P51G-m4 and I34L are equally stable, with apparent $\mathrm{T}_{\mathrm{m}}$ of 58 and 57.8 respectively. In 232 contrast, the I34Y and I34K mutants were less thermostable than P51G-m4 as shown by apparent $233 \mathrm{~T}_{\mathrm{m}}$ values of 54.7 and $47^{\circ} \mathrm{C}$ respectively. Substituting a hydrophobic residue with a basic aliphatic 
234 amino acid (lysine) has a large destabilizing effect, while aromatic and polar tyrosine is better

235 tolerated. The trend for thermostability is P51G-m4 I34L > I34Y > I34K (Fig S1).

239 the samples for 72-hour (Patsalo et al., 2011). The results align with the thermal denaturation

240 results: P51G-m4 is the most stable to denaturant, followed by I34L, I34Y and I34K (Fig S2). The

241 data were analyzed as described in methods to extract $\Delta \mathrm{G}_{\mathrm{H} 20}$ values and $\mathrm{C}_{\mathrm{m}}$ values of for P51G-

$242 \mathrm{~m} 4, \mathrm{I} 34 \mathrm{~L}, \mathrm{I} 34 \mathrm{Y}$ and $\mathrm{I} 34 \mathrm{~K}$ of $3.0,2.94,2.91$ and $2.38 \mathrm{kcal} / \mathrm{mol}$ and of $1.45,1.39,1.13$ and $0.68 \mathrm{M}$

243 respectively (Table 2).

Table 2. The $\Delta \mathrm{G}_{\mathrm{H} 2 \mathrm{O}}$ and $\mathrm{C}_{\mathrm{m}}$ values for $\mathrm{CV}-\mathrm{N}$ mutants obtained from chemical denaturation experiments

\begin{tabular}{ccc}
\hline Variants & $\Delta \mathbf{G}_{\mathbf{H} 2 \mathbf{O}}(\mathbf{K c a l} / \mathbf{m o l})$ & $\mathbf{C}_{\mathbf{m}}(\mathbf{M})$ \\
\hline P51G-m4 & $3.01 \pm 0.047$ & $1.46 \pm 0.019$ \\
P51G-m4-I34L & $2.95 \pm 0.077$ & $1.39 \pm 0.009$ \\
P51G-m4-I34Y & $2.91 \pm 0.157$ & $1.13 \pm 0.017$ \\
\hline P51G-m4-I34K & $2.40 \pm 0.124$ & $0.68 \pm 0.015$ \\
\hline
\end{tabular}

247 the mutants for dimannose were assessed by isothermal titration calorimetry (ITC) (Fig S3),

248 obtaining $K_{d}$ values listed in Table 1. We found that I34Y binds dimannose with tightest affinity

249 of all the mutants tested and showed a two-fold improvement over P51G-m4, with $\mathrm{K}_{\mathrm{d}}$ of $64 \mu \mathrm{M}$

250 as compared to $117 \mu \mathrm{M}$. I34L showed a slight decrease in binding with a $\mathrm{K}_{\mathrm{d}}$ of $148 \mu \mathrm{M}$. No binding

251 was observed for I34K. Thermodynamic values extracted from ITC experiments (Table 1) also 
252 show that the change in binding is related to changes in entropy, particularly for I34Y. These data

253 support the hypothesis that I34 modulates the conformational dynamics of the pocket.

To glean more information on the effect of the mutations, we determined the X-ray

256 structure of the I34Y mutant with and without dimannose and compared it to the template protein

257 P51G-m4 by superimposing the structures. The fold is highly conserved (Fig 2) as shown by root

258 mean squared distance (RMSD) of 0.16 and $0.20 \AA$ with bound and unbound I34Y, respectively,

259 and tyrosine is well tolerated at position I34. The binding pocket region is also structurally

260 conserved compared to P51G-m4. Analysis of the polar contacts with dimannose compared

261 between P51G-m4 and I34Y (Fig 2B) shows that both proteins make an identical number of

262 hydrogen bonds (11) with the ligand, indicating a conserved binding pose for dimannose. These

263 observations suggest that the increase in binding affinity of I34Y towards dimannose might be

264 mediated by equilibrium dynamics, which are not captured by the crystal structure. This hypothesis

265 is supported by the changes in entropy compensation measured experimentally (ITC) in dimannose

266 binding by P51G-m4 (negative TDS) and I34Y (positive TDS). 


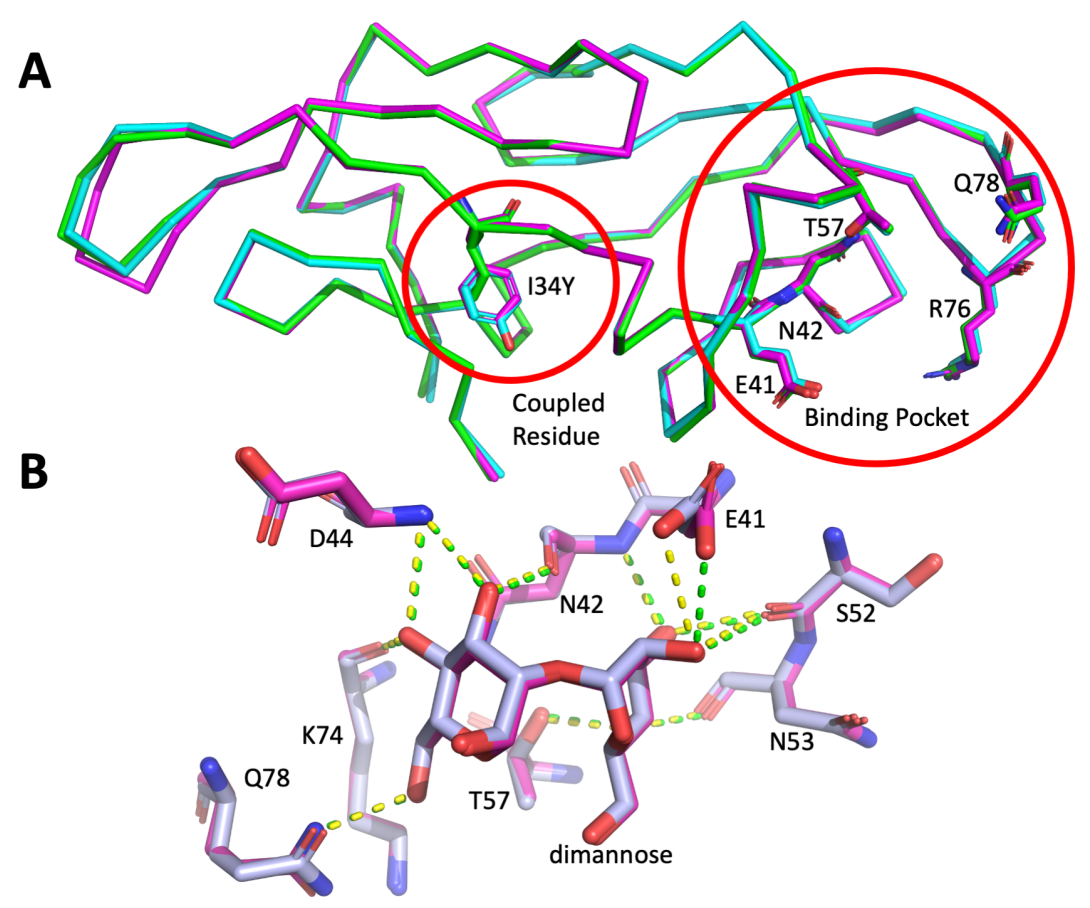

Fig 2 A) The crystal structures of I34Y (bound in magenta and unbound in cyan) and its template protein P51G-m4 (green) are superimposed. B) Overlay of bound structures of I34Y (magenta) and P51G-m4 (grey) (RMSD $0.15 \AA$ ); dashed lines depict polar interactions with dimannose.

To gather atomic level detail on how the substitutions on I34 dynamically modulate the 270 binding affinity, we performed two microsecond molecular dynamics simulations for each variant 271 and P51G-m4 to obtain equilibrium dynamics (See Methods for details of the simulations). Our 272 earlier work showed that residues N53 and N42 play a key role in regulating the binding pocket 273 accessibility to the glycans: A hydrogen bond between the amide hydrogen of N42 and carbonyl 274 oxygen of $\mathrm{N} 53$ forms a closed pocket, hindering glycan accessibility, whereas the loss of this 275 hydrogen bond leads to an open pocket (Li et al., 2015). Comparison of open and closed 276 conformations based on this hydrogen bond shows that I34Y variant samples the open binding 277 pocket more often than P51G-m4 (Fig S4). 
Another compelling evidence differentiating I34 variants from P51G-m4 is the change in 280 their binding pocket volumes. The MD simulations were analyzed by POVME pocket volume 281 calculation tool (Wagner et al., 2017). The calculated pocket volumes for I34Y, I34K and P51G$282 \mathrm{~m} 4$ were converted into frequencies to obtain probability distribution of the pocket volumes (Fig 283 3A), revealing that $\mathrm{I} 34 \mathrm{Y}$ variant samples a more compact pocket volume compared to P51G-m4.

284 If the pocket is too small or too large dimannose cannot maximize its interaction with the protein, 285 and a compact conformation enables dimannose to easily make the necessary hydrogen bond 286 interactions with the protein. This optimum pocket volume sampled by I34Y may also explain the 287 different binding energetics observed by ITC, in which a positive entropy change upon binding 288 compensates for the loss in enthalpy compared to P51G-m4. Pocket volume analysis reveals a 289 larger value for $\mathrm{I} 34 \mathrm{~K}$ compared to P51G-m4, suggesting that this mutant cannot accommodate the 290 necessary interactions with the dimannose resulting in loss of binding. We applied the same pocket 291 volume calculation to the X-ray structures of P51G-m4 and I34Y variant, and we found volumes 292 of $141 \AA^{3}$ and $114 \AA^{3}$ for P51G-m4 and I34Y, respectively in the unbound forms (Fig 3B). These 293 volumes from structures correlates well with the mean volumes from MD simulation trajectories, 294 hinting that the variants modulate the conformational dynamics of binding pocket. Overall, in 295 agreement with ITC analysis, the results show a shift in native state ensemble upon allosteric 296 mutations, modifying the glycan-CV-N interactions. While our conformational dynamics analysis 297 suggest that the shift of the native ensemble towards a smaller pocket volume upon I34Y mutation 298 can explain the decrease in the entropic cost of binding, observed as positive entropy change in 299 ITC, the drastic decrease in enthalpic component in ITC fit is further investigated by analyzing 300 binding energetics of the docked poses obtained through performing dimannose docking on 2000 301 different conformations sampled accordingly from the binding pocket volume distributions. This 
analysis shows that the small volume restrict accessibility to the side-chain conformations of binding residue R76 in I34Y variant yielding different hydrogen bond patterns with the dimannose

304 (Fig S5), suggesting a loss in enthalpic contribution.

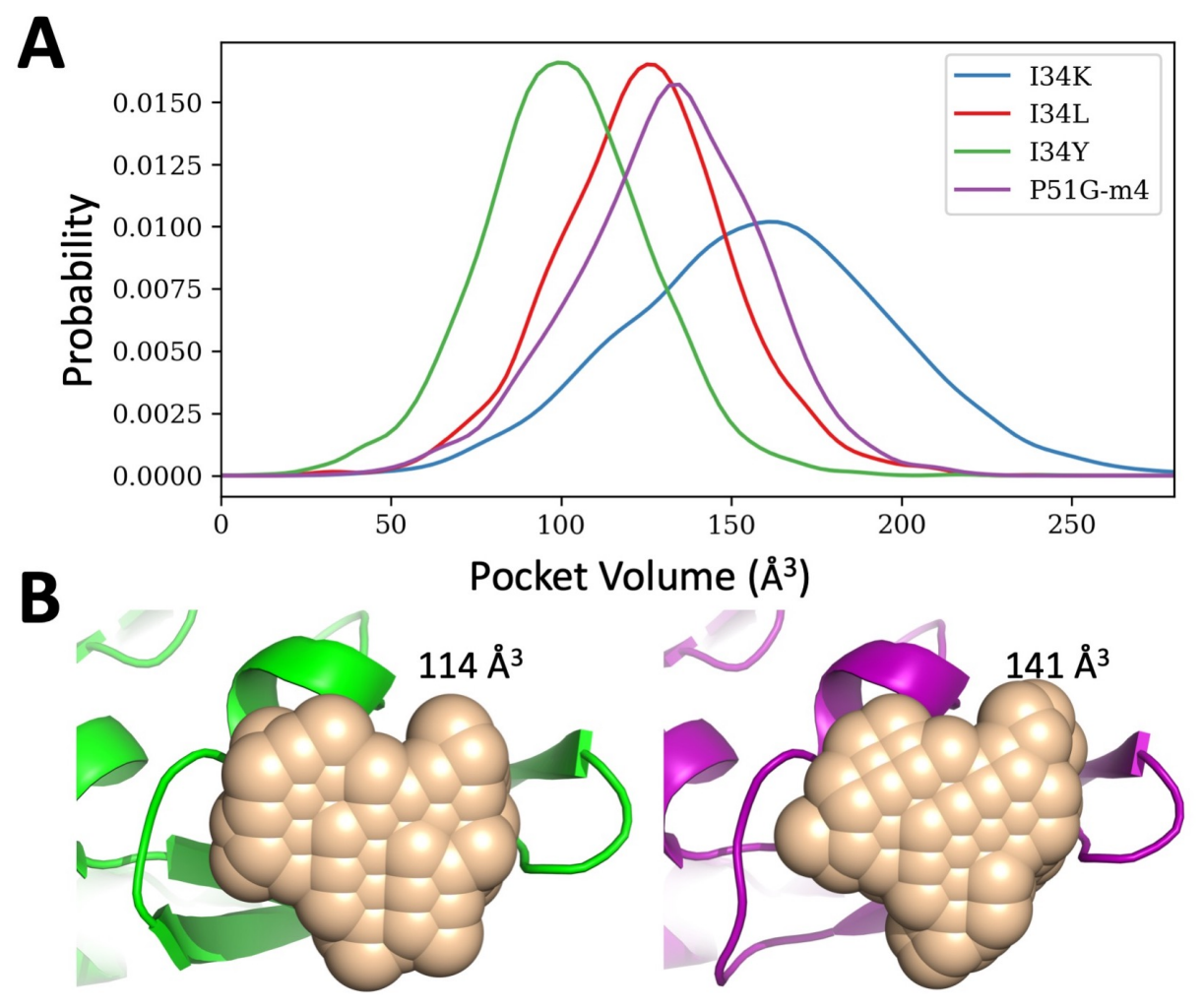

Fig 3 A) Probability distribution of the pocket volume analyses obtained from MD simulation trajectories. Comparison between different variants presents that I34Y stays in an optimum volume more than others favoring a suitable pocket for binding. P51G-m4 and I34L variant sample similar pocket volumes. but I34K variant has a larger pocket volume compared to others. B) Pocket volume comparison of the domain B of solved structures for P51G-m4 (purple colored structure on right) and I34Y variant (green colored structure on left). 
Substitutions of I34 modulates conformational ensemble leading to change in glycan binding affinity

311 of a protein by modulating the native state ensemble through mutations of different positions while

312 keeping the 3-D structure conserved (Campitelli et al., 2020a; Kuriyan and Eisenberg, 2007; Li et

313 al., 2015; Liu and Nussinov, 2017; Modi and Ozkan, 2018; Risso et al., 2018; Tripathi et al., 2015;

314 Woodrum et al., 2013). Furthermore, the shift in native state ensemble is reflected in equilibrium

315 dynamics and the flexibility profiles obtained by DFI capture this shift. DFI profiles that show the

316 flexibility/rigidity of each position withing the 3-D structure correlate well with the changes in

317 function throughout evolution. We observed a similar pattern of conservation of structure yet

318 change in function in our designed CV-N I34 variants, thus we further analyzed the DFI profiles

319 of I34 variants. We clustered the DFI profiles of I34 mutants and P51G-m4 using principal

320 component analyses (PCA) and compared with a 2-D map of these mutants in which melting

321 temperature and $\mathrm{K}_{\mathrm{d}}$ are used as reaction coordinates (Fig 4). The 2-D map reveals I34L, P51G-m4,

322 and I34Y clustered together, particularly I34L, P51G-m4, is the closest in this 2-D map while I34K

323 is on the other corner of the map based on stability and binding affinity (Fig 4A). Interestingly, the

324 dendrogram constructed based on the DFI similarities of these proteins using PCA analysis

325 captures biophysical properties (Fig 4B). The clustered DFI profiles put P51G-m4 and I34L variant

326 under the same branch, suggesting their dynamics are very similar; thus, function associated with

327 these dynamics should also be similar. I34Y is also under the same main cluster with the other two

328 proteins that also exhibit dimannose affinity, yet it is in a different branch. This presents that I34Y

329 exhibits slightly different DFI profile suggesting that slight modification in its DFI profile may be 
associated with enhanced binding affinity. On the other hand, I34K is under a separate branch,

331 showing that its dynamics is completely different than others. Therefore, the DFI clustering mirrors

332 the clusters observed in the functional 2-D map, implying a correlation between changes in

333 dynamics and changes in fitness. This is in agreement with our previous studies, where

334 substitutions on DARC spots modulates flexibility profiles to adapt to a new environment

335 (Campitelli et al., 2020b; Kumar et al., 2015b).
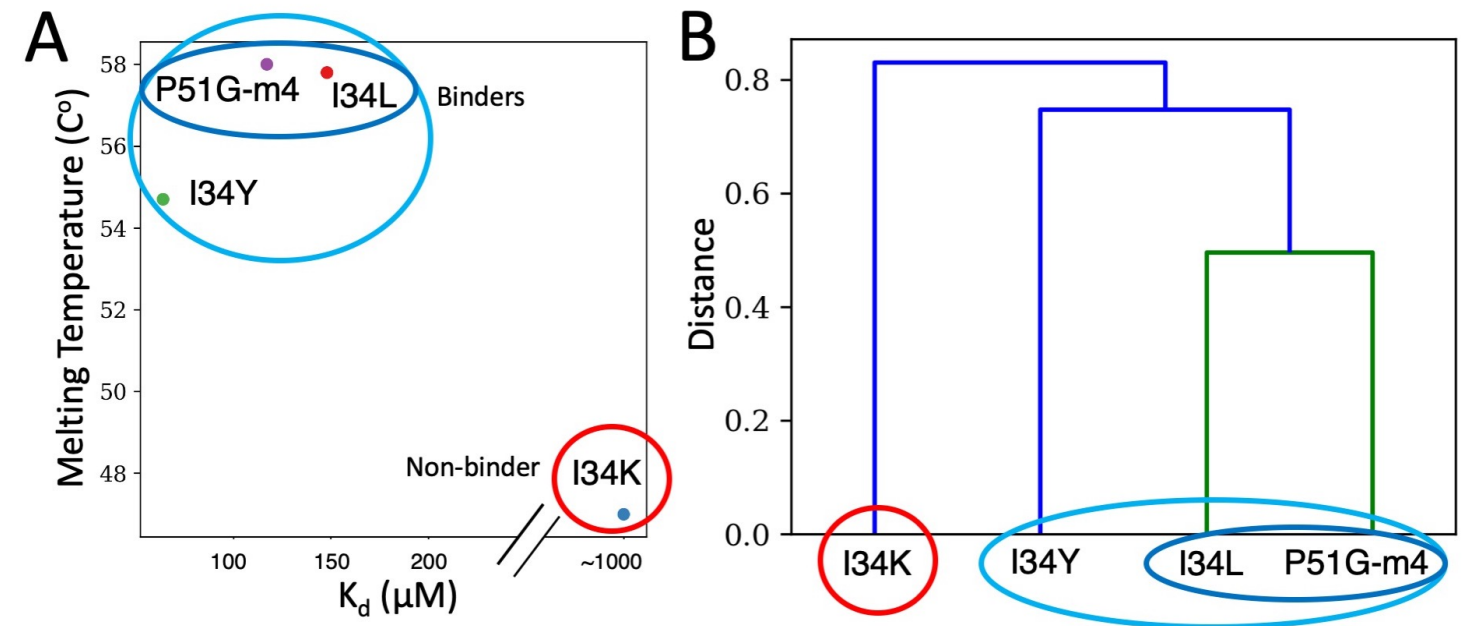

Fig 4 A) $K_{d}$ and Melting temperature of P51G-m4 and its variants plotted together B) PCA clustering on the first two principal components of the DFI profiles of P51G-m4 and its variants as a dendrogram.

As clustering of mutants using their DFI profiles aligns with their biophysical

339 characterization, we further investigated the change in DFI profiles of the binding sites with respect

340 to the P51G-m4 for each mutant (Fig 5A). This analysis reveals that change in DFI values of the

341 binding site position T57 exhibits distinct variation among I34 mutants. Position T57 in the case

342 of deleterious mutant (abolishing binding) I34K, exhibits higher flexibility, suggesting a higher 
343 entropic penalty for binding interactions. The flexibility profile of I34L variant in position T57 is

344 similar to that of P51G-m4. Accordingly, they show similar binding affinities. In contrast, T57

345 becomes much more rigid for I34Y mutant, suggesting that a decrease in the entropic cost can

346 contribute to the binding affinity enhancement of this mutant. In short, the change in flexibility of

347 the position T57 correlates well with the change in binding affinities of these different mutants.

348 This is indeed not a surprising observation, since our previous study of single point mutations of

349 the binding pocket residues have shown that T57 provides one of the key side chain interactions

350 with dimannose.

The rigidification of $\mathrm{T} 57$, a position far from the mutation location $\mathrm{I} 34$, is a compelling

353 evidence that the distal mutation is allosterically controlling the binding site dynamics. We further

354 computed the network of interactions that connects the residue position 34 to 57 and investigated

355 whether distinct pathways emerge after mutation of I34Y. We analyzed the hydrogen bond

356 networks, particularly computed the possible network of hydrogen bonds creating pathways from

35734 to 57 using the sampled snapshots in our MD trajectories (Fig S6). This analysis presents a

358 unique pathway from 34 to 57 by first forming a new hydrogen bond between the side chain

359 oxygen of the Tyrosine of residue 34 and the nitrogen of the Tyrosine of residue 100 in I34Y

360 variant. Furthermore, a second pathway is also found which is sampled much more frequently in

361 I34Y variant strengthening the communication between position 34 and 57 . Thus, both pathways

362 may contribute to the rigidification of T57. We also analyzed the structures from MD clustered

363 with highest percentage based on alpha carbon RMSD for I34Y and P51G-m4 and compared the

364 hydrogen bond interactions of T57 and its neighboring residues. The closest neighbors of T57;

365 positions I55, E56, and C58 conserved their hydrogen bond interactions with their surrounding 
residues between P51G-m4 and I34Y. On the other hand, T57 is observed to make an extra

367 hydrogen bond interaction in I34Y compared to P51G-m4 (Fig 5B), suggesting that enhancement

368 in hydrogen bond networking of T57 in I34Y leads to rigidification of this position in equilibrium

369 dynamics.

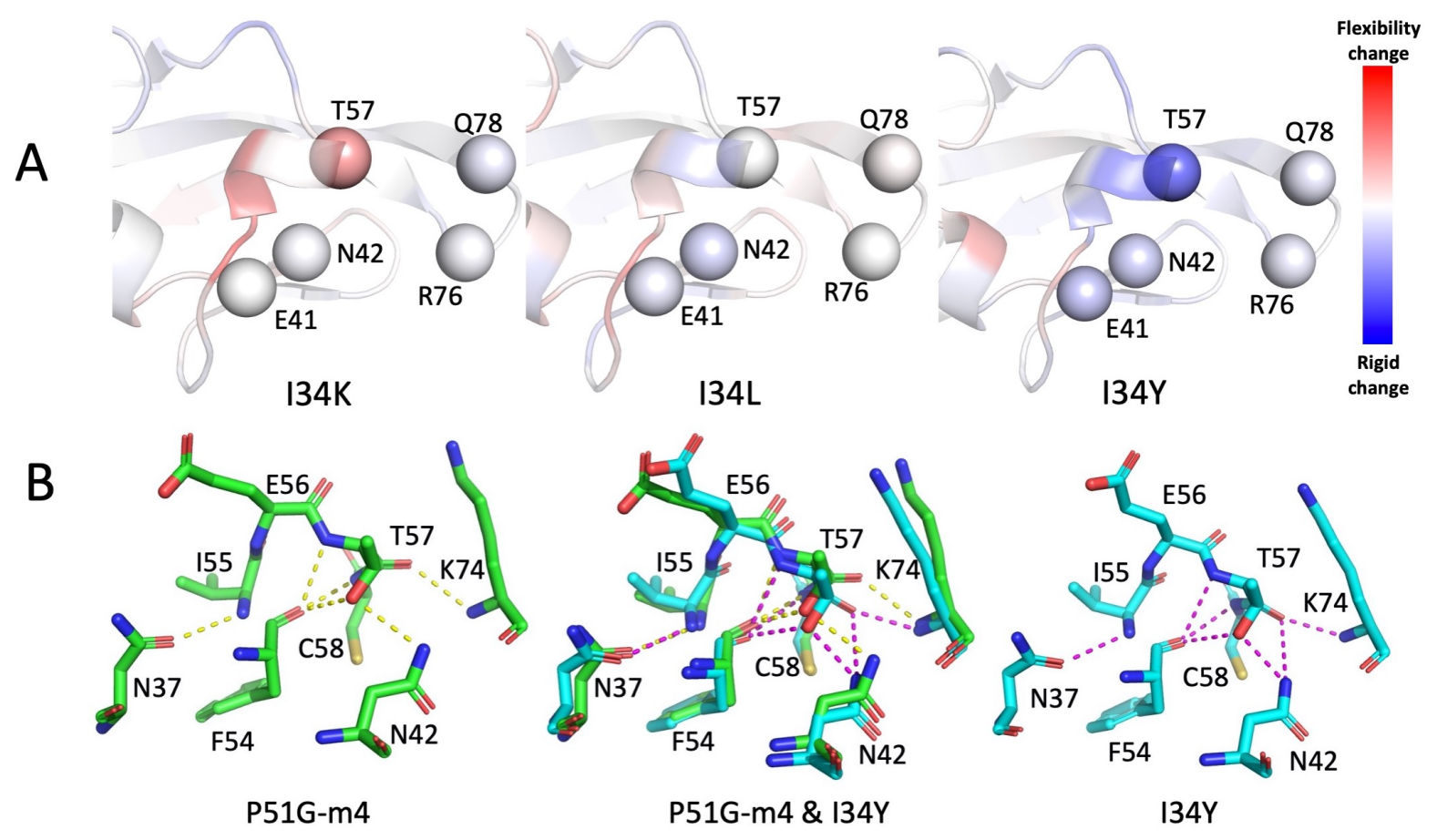

Fig 5 A) Change in DFI of I34K, I34L, and I34Y relative to P51G-m4 are projected on structure.

Residues E41, N42, and T57 rigidifies on I34Y compared to P51G-m4. B) Hydrogen bonding interactions of residues I55, E56, T57, and C58 are shown for P51G-m4 and I34Y variant. The rigidification on location T57 can be understood by its neighboring interactions. Compared to P51G-m4, the I34Y variant makes an extra hydrogen bond making it a more rigid residue. This rigidity is reflected on the binding as T57 makes direct interaction with dimannose. 
Taken together, these analyses are in agreement with experimental observation of

373 modulation of entropy of binding to alter affinity where mutation on I34 distally regulates the

374 binding pocket dynamics particularly the position T57, leading to a change in binding energies.

376 Conclusion et al., 2019) yet predicting which sites are involved through computational analysis is challenging. Indeed, many of these sites are identified only after rounds of directed evolution in protein design

383 (Barrientos et al., 2006; Keeffe et al., 2011; Romero and Arnold, 2009; Yuan et al., 2005). Here,

384 we showed that it is possible to identify these distal sites and fine-tune the binding interactions by 385 integrating long range dynamic coupling with coevolution principles. Coupling DCI approach

386 which selectively identify distal positions that are much more sensitive to the fluctuations of 387 functional sites with evolutionary inference of mutational landscape allowed us to identify mutants 388 that may modify function. We first computationally screened impact of these mutants via Adaptive 389 -BP dock and distinguished beneficial variants from those deleterious. Specifically, we observed 390 that substitutions on these distal sites altered equilibrium dynamics, while conserving the 3-D fold 391 and the binding motif, as also highlighted as change in change in entropic contribution in ITC 392 binding analysis. 
Our new approach that couples evolutionary inferences with long-range dynamic communications within the 3-D network of interactions paves the way to incorporating distal

396 mutants into protein design.

\section{Adaptive BP-Dock}

Adaptive backbone perturbation docking, Adaptive BP-Dock in short, allows us to model the interaction between CV-N and glycans in silico (Bolia and Ozkan, 2016). Adaptive BP-dock combines the complex simulation of backbone flexibility of a protein into Rosetta's ligand docking application (Davis and Baker, 2009). The common restriction in docking is the implementation of flexibility of receptor and ligand (Davis et al., 2009; Davis and Baker, 2009; DeLuca et al., 2015;

407 Meiler and Baker, 2006). Rosetta included the flexibility of ligand in their monte-carlo sampling 408 approach but lacking full receptor flexibility. This high order challenge is overcome by utilizing 409 Perturbation Response Scanning (PRS) to compute backbone changes during docking (Atilgan and 410 Atilgan, 2009; Bolia et al., 2014b, 2014a; Ikeguchi et al., 2005). This procedure also allows the 411 modeling of transition from an unbound state to a bound state (Bolia and Ozkan, 2016). The

412 computational cost of sampling is reduced by using a coarse-grained approach employing Elastic

413 Network Model (ENM) leading to an efficient way of computing backbone perturbations,

414 mimicking the ligand interacting with receptor (Atilgan et al., 2001, 2010; Atilgan and Atilgan, 415 2009). 
418 unbound conformation of CV-N. The perturbed pose of the protein is calculated using PRS. The

419 structure is then minimized, and the side chains are added at this step. The glycan is docked to the

420 minimized structure using RosettaLigand algorithm. Rosetta samples bound conformations using

421 a knowledge based potential function and calculates bound pose energies. The lowest energy

422 docked pose is selected and feed back to perturbation step, and the same procedure is followed

423 iteratively until a convergence is reached. At the end of each iteration the lowest energy docked

424 pose is taken and binding score is calculated using an empirical scoring function X-score. X-score

425 has shown to provide higher correlations with experimental results (Wang et al., 2002). The flow

426 of the algorithm is shown in Fig 6.

Adaptive BP-Dock Docking Scheme

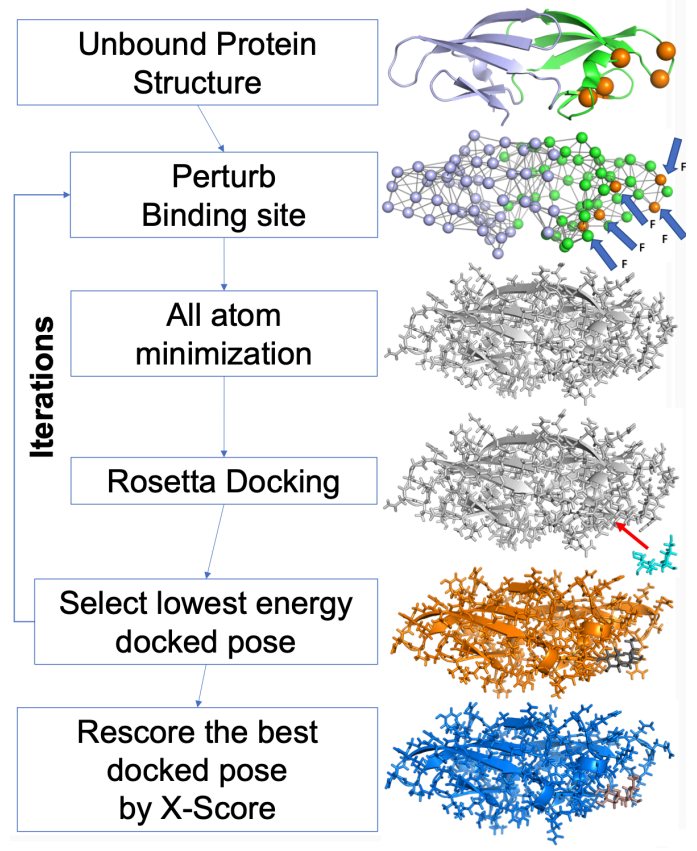

Fig 6 The flow of Adaptive BP-Dock. Starting from an unbound structure the backbone atoms are perturbed using PRS and perturbed pose is fed into minimization. The minimized 
conformation is docked with the glycan using RosettaLigand docking approach. The lowest scored docked pose is selected and used for next iteration step until convergence is established. For every lowest energy docked pose from an iteration a new binding score is reevaluated using X-score empirical scoring function (Wang et al., 2002).

Adaptive BP-Docks iterative algorithm ensures the sampling does not stuck in a local minimum and reaches a global minimum. The challenge of unbound/bound modeling is solved

431 using the iterative approach as the conformations are led towards a bound pose with the help of 432 PRS.

\section{Molecular Dynamics (MD)}

437 (Abraham et al., 2015; Spoel et al., 2005). For each simulation the all-atom system is parametrized 438 with CHARMM36 force field and explicit water model TIP3P. The solvation box is set to be 439 minimum $16 \AA$ from protein edge. The system is then neutralized by potassium ions to sustain 440 electroneutrality and minimized with steepest descent for 10000 steps. A short-restrained 441 equilibrium is conducted in the constant number of particles, pressure, and temperature ensemble 442 (NPT) for $5 \mathrm{~ns}$ using the Berendsen method at $300 \mathrm{~K}$ temperature and 1 bar pressure. NPT 443 production trajectories were performed with Nose-Hoover and Parrinello-Rahman temperature 444 and pressure coupling methods for $2 \mu \mathrm{s}$ at $300 \mathrm{~K}$ and 1 bar. For all cases periodic boundary 445 conditions and particle-mesh Ewald (PME) with interaction cutoff of $12 \AA$ is employed with 446 Gromacs version 2018.1. 
450 DFI is residue position specific metric that can measures the resilience of a given positions to the

451 force perturbations. It is the fluctuation response of a residue relative to the gross fluctuation 452 response of the protein (Kumar et al., 2015b; Larrimore et al., 2017). DFI calculates residue 453 response due to a perturbation by utilizing covariance matrices.

$$
[\Delta \mathbf{R}]_{3 N x 1}=[\mathbf{H}]_{3 N x 3 N}^{-1}[\mathbf{F}]_{3 N x 1}
$$

$$
D F I_{i}=\frac{\sum_{j=N}^{N}\left|\Delta R^{j}\right|_{i}}{\sum_{i=1}^{N} \sum_{j=1}^{N}\left|\Delta R^{j}\right|_{i}}
$$

461 derivatives of potentials. The inverse of Hessian matrix, $\boldsymbol{H}^{-1}$, contains residue covariances, and 462 interpreted as a covariance matrix. The covariance matrices can be gathered from MD simulations, 463 and also by using Elastic Network Model (ENM) of a protein. In this study, MD covariance 464 matrices have been utilized to incorporate residue interactions accurately. 
residues exhibiting low DFI values are crucial as information gateways. While they do not exhibit

469 high residue fluctuation to the perturbations, they quickly transfer the perturbation information to

470 other parts, thus they are in control of collective motion of the protein. A change in low DFI

471 positions (i.e., a mutation) will lead to a transformation in the communication grid and majority of

472 disease-associated (i.e. function altering mutations) are often observed in hinge, low DFI sites

473 (Butler et al., 2015; Gerek et al., 2013; Kumar et al., 2015a). The substitution on these site usually

474 alters catalytic activity or binding interaction (i.e., glycans) by modulating equilibrium dynamics

475 (Campitelli et al., 2020a).

476

477 Dynamic Coupling Index (DCI)

Dynamic Coupling Index (DCI) exploits the same framework of DFI (Campitelli et al., 2020a; Larrimore et al., 2017). DCI utilizes the residue response fluctuation upon random force perturbation at a specific residue positions to investigate residues that exhibit long-range coupling to each other. In DCI approach, a unit force is applied on functional residues (i.e., binding site residues) one by one and responses of all other residues are calculated.

$$
D C I_{i}=\frac{\sum_{j=N_{\text {Functional }}}^{N_{\text {Functional }}}\left|\Delta R^{j}\right|_{i} / N_{\text {Functional }}}{\sum_{j=1}^{N}\left|\Delta R^{j}\right|_{i} / N}
$$

With DCI scheme the residues with high response (high DCI score) indicates high long 
490 importance in how forces propagate through amino acid chain network on a binding event. Some

491 of the coupled residues are far from the binding site but still encompass modulation capabilities

492 over binding pocket.

493

494 Informing dynamics from coevolution

Coevolutionary data paves the way to assessing 3-D structural contacts by utilizing

497 available sequence information (Hopf et al., 2018; Marks et al., 2012; Morcos et al., 2014).

498 Sequence information is more abundant compared to resolved protein structures. Exploiting the

499 sequence information, primary contacts comparable to realistic structural contacts can be

500 calculated and a contact matrix is formed. The accuracy of these contact maps is proved to be

501 valuable in protein folding studies (Kryshtafovych et al., 2019; Morcos et al., 2011; Wang et al.,

502 2016). Evolutionary coupling (EC) analysis is used to collect information on how much two

503 residues in a protein sequence is in close proximity in 3-D structure. EC scores could be calculated

504 by many different statistical approaches. In this study EC information is gathered by using

505 RaptorX, EVcouplings, and MISTIC webservers (Hopf et al., 2019; Simonetti et al., 2013; Wang

506 et al., 2017). While the limitation of these methods emerges from sequence homolog availability

507 of a protein in multiple sequence alignment (MSA), RaptorX uses a deep neural network

508 leveraging joint family approach, combining multiple ortholog protein families sharing similar

509 function and phylogeny, to infer possible contacts. This method is proven to produce high accuracy

510 in contact prediction compared to others (Wang et al., 2017). However, for a given MSA

511 containing enough homolog sequences other methods are also strong in predicting spatial contacts.

512 EVcouplings approach uses Direct Information (DI) to calculate coevolutionary couplings. DI 
513 metric is a modified mutual information (MI) score considering consistency between pairwise

514 probabilities and single amino acid frequencies (de Juan et al., 2013; Morcos et al., 2011).

515 Nonetheless, MI, a global approach compared to local DI metric, is accurate in capturing true

516 contacts, while entangling indirect contacts from direct contacts. MISTIC web server has taken

517 advantage of MI to calculate coevolutionary couplings (Dunn et al., 2008; Gouveia-Oliveira and

518 Pedersen, 2007; Simonetti et al., 2013). In their MI method they introduced a correction term to

519 MI to surpass the low statistics gathered with an MSA containing limited number of sequences.

520 This approach is very useful in cases where certain homologs are rare and MSA of these homologs

521 have multiple gaps in their alignments. All of these methods are employed in this study to achieve

522 high accuracy predictions in finding residue couplings.

\section{Mutant proteins cloning, expression and purification}

The genes for mutants (I34Y, I34K, and I34L) were generated by applying mutagenic

527 primers to P51G-m4-gene sequence and amplifying by PCR. The constructs were subsequently

528 cloned in pET26B vector between NdeI and XhoI sites and transformed in BL21(DE3) for

529 expression and purification. The proteins were expressed from a $10 \mathrm{ml}$ starter culture in LB broth

530 overnight at $37^{\circ} \mathrm{C}$, inoculated into $1 \mathrm{~L} \mathrm{LB}$ medium. The culture was induced with $1 \mathrm{mM}$ isopropyl

531 thiogalactoside (IPTG) when OD reached 0.6 and grown for another 6-8 hours. The cells were

532 harvested by centrifugation, lysed in 6M Guanidine hydrochloride at $\mathrm{pH}$ 8.0, and sonicated for 10

533 minutes. The supernatant recovered after centrifugation was used to purify proteins with GE

534 HisTrap HP column (GE Healthcare Bio-Sciences, Piscataway, NJ) and a Bio-Rad EconoPump

535 (Bio-Rad, Richmond, CA) under denaturing conditions. In brief, the proteins were loaded on the 
536 column in $\mathrm{Gu}-\mathrm{HCl}$ buffer, which was buffer exchanged by $8 \mathrm{M}$ Urea buffer. The nonspecific

537 proteins were washed out by $4 \mathrm{M}$ urea and $20 \mathrm{mM}$ imidazole buffer, $\mathrm{pH} 8.0$ and eluted with $2 \mathrm{M}$

538 Urea and 200mM imidazole, pH 8.0 buffer before putting it for overnight dialysis against $10 \mathrm{mM}$

539 Tris $\mathrm{pH} 8.0$ and $100 \mathrm{mM} \mathrm{NaCl}$ buffer. The buffer was changed once during the night. The refolded

540 protein was concentrated and re-purified to isolate the monomeric species by size exclusion

541 chromatography using Sephadex 75 10/300 column on Agilent's Infinity 1260 system. The gel

542 filtered protein was finally used for all the experiments.

\section{CD spectroscopy and T-melts}

In CV-N family proteins, thermodynamic parameters like free energy of unfolding,

547 enthalpy and entropy cannot be extracted by thermal denaturation because the transition from

548 folded to unfolded state is non-reversible (Patsalo et al., 2011), therefore melting temperatures are

549 used. Far-UV CD spectra were recorded on a Jasco J-815 spectropolarimeter equipped with a

550 thermostatic cell holder, PTC 424S. Spectra were measured from 250 to $200 \mathrm{~nm}$, using a scanning

551 speed of $50 \mathrm{~nm} / \mathrm{min}$ and a data pitch of $1.0 \mathrm{~nm}$ at $25^{\circ} \mathrm{C}$. Samples concentration was approximately

$55215 \mu \mathrm{M}$ in $10 \mathrm{mM}$ Tris, $\mathrm{pH} 8.0$ and $100 \mathrm{mM} \mathrm{NaCl}$. For thermal denaturation experiments, the

553 melting profile was monitored at $202 \mathrm{~nm}$ from $25{ }^{\circ} \mathrm{C}$ to $90{ }^{\circ} \mathrm{C}$. The data points were plotted and

554 fitted in Origin8.5 software to get apparent Tm. 


\section{Isothermal titration calorimetry (ITC)}

ITC was performed at the Sanford-Burnham Medical Research Institute Protein Analysis

562 Facility using ITC200 calorimeter from Microcal (Northampton, MA) at $23{ }^{\circ} \mathrm{C} .2 .0 \mu 1$ aliquots of

563 solution containing between $3 \mathrm{mM}$ and $10 \mathrm{mM}$ Man2 were injected into the cell containing between

5640.057 , and $0.11 \mathrm{mM}$ protein. 19 of $2.0 \mu 1$ injections were made. The experiments were performed

565 in $10 \mathrm{mM}$ Tris, $100 \mathrm{mM} \mathrm{NaCl}, \mathrm{pH} 8.0$ buffer. ITC data were analyzed using Origin software

566 provided by Microcal.

567

\section{Chemical denaturation experiments}

571 tryptophan fluorescence on Cary Eclipse instrument (Varian). $10 \mu \mathrm{M}$ of protein samples were

572 incubated with increasing concentrations of Guanidine hydrochloride in the range of 0-6 $\mathrm{M}$ in 50

$573 \mathrm{mM}$ Tris $\mathrm{pH} 8.0$ buffer for 72 hours at $25^{\circ} \mathrm{C}$. The emission spectra for the same were recorded by

574 keeping the excitation wavelength at $295 \mathrm{~nm}$ and bandwidth of $1 \mathrm{~nm}$. A ratio of fluorescence at

575330 and $360 \mathrm{~nm}\left(\mathrm{I}_{330} / 360\right)$ was plotted at respective $\mathrm{Gu}-\mathrm{HCl}$ concentrations and the data points were

576 fit to following sigmoidal equation to obtain $\mathrm{C}_{\mathrm{m}}$.

$$
y=\mathrm{A} 2+\frac{\mathrm{A} 1-\mathrm{A} 2}{1+\mathrm{e}^{(\mathrm{x}-\mathrm{x} 0) / \mathrm{dx}}}
$$

578 Where, A1 and A2 are the initial and final 330/360 ratios and $\mathrm{x} 0$ is the concentration of $\mathrm{Gu}-\mathrm{HCl}$, 579 where $y=(\mathrm{A} 1+\mathrm{A} 2) / 2$, or the point where $50 \%$ of the population is unfolded. It is also denoted as $580 \mathrm{C}_{\mathrm{m}}$. 
The denaturation curve was used to calculate the free energy of the protein in the absence of denaturant $\left(\Delta \mathrm{G}_{\mathrm{H} 2 \mathrm{O}}\right)$. Fraction unfolded $\left(f_{\mathrm{U}}\right)$ was calculated using the following formula:

$$
f_{\mathrm{U}}=\left(y_{\mathrm{F}}-y_{\mathrm{obs}}\right) /\left(y_{\mathrm{F}}-y_{\mathrm{U}}\right)
$$

584 where $f_{\mathrm{U}}$, is the fraction unfolded, $y_{\mathrm{F}}$ is the value when there is no denaturant, $y_{\mathrm{obs}}$ is the value at 585 each position and $y_{\mathrm{U}}$ is the value for unfolded protein. Since $f_{\mathrm{U}+} f_{\mathrm{F}}=1$, The equilibrium constant,

$586 \mathrm{~K}$, the free energy change can be calculated using

Where $\mathrm{R}$ is the gas constant whose value is $1.987 \mathrm{cal} / \mathrm{mol} . \mathrm{K}$ and $\mathrm{T}$ is the temperature of incubation, which was $25^{\circ} \mathrm{C}$. The value of $\Delta \mathrm{G}$ is linear over a limited range of $\mathrm{Gu}-\mathrm{HCl}$. The linear fit over that range was extrapolated to obtain $\Delta \mathrm{G}_{\mathrm{H} 2 \mathrm{O}}$.

Crystallization and structure determination

I34Y was purified as discussed above and the monomeric gel filtered protein was

597 concentrated to $8 \mathrm{mg} / \mathrm{ml}$. We got the crystals in $2 \mathrm{M}$ ammonium sulphate and $5 \%$ (v/v) 2-propanol

598 after screening it in Index HT screen from Hampton Research. The protein crystals were 599 reproduced using same condition in hanging drop method. For protein crystals with dimannose,

600 the crystals were incubated in 1.2 fold molar excess of dimannose. Single needle-like crystals were 601 picked up and cryo-preserved in $25 \%$ glycerol before freezing them for data collection at 602 Synchrotron ALS, beamline 8.2.1. Single crystal diffraction was measured at wavelength of 0.999 603 A with ADSC quantum $315 \mathrm{r}$ detector. The data were evaluated to resolution of $1.25 \mathrm{~A}$. The data 
604 acquired was indexed using XDS and scaled by the aimless package from CCP4i program suite.

605 The structural coordinates and phase were determined by molecular replacement using 2RDK PDB

606 code. The structure of I34Y of CV-N is deposited under PDB accession code 6X7H. The structure

607 was further refined in Coot.

608

\section{Acknowledgements}

610 S.B.O. acknowledges support from the Gordon and Betty Moore Foundations and National

611 Science Foundation (Award: 1715591 and 1901709). G.G. and S.B.O. is grateful for the grant

612 1R21CA207832-01.

613

\section{Competing interests}

615 No competing interests declared.

616

\section{Supplementary Data}

Table S1. DFI, DCI, RaptorX, Evcoupling, and MISTIC metrics are used to identify critical residues both rigid, distal from binding site, allosterically coupled and coevolved with binding sites (Supplementary file 1 table 1). The analyses pointed out four residues matching the rigidity (DFI score less than 0.2), allosteric coupling (DCI score higher than 0.7), coevolution (RAPTORX, MISTIC, and EVCOUPLING all higher than 0.6) and distance (Minimum distance to binding site higher than $10 \AA$ ) criteria. Only residue I34 out of four residues satisfy a far 
distance measure (Both minimum and average distance to binding site higher than $15 \AA$ ). We selected residue $\mathrm{I} 34$ for further analyses.

\begin{tabular}{|c|c|c|c|c|c|c|c|}
\hline \multirow{2}{*}{ Residue } & \multirow{2}{*}{$\begin{array}{c}\text { DFI } \\
\text { Score }\end{array}$} & \multirow{2}{*}{$\begin{array}{c}\text { DCI } \\
\text { Score }\end{array}$} & \multicolumn{2}{|c|}{ Distance from Binding Sites $(\mathbf{\AA})$} & RAPTORX & MISTIC & EVCOUPLING \\
Score & Minimum & Average & Score & Score \\
\hline 34 & 0.17 & 0.71 & 16 & 22 & 0.65 & 0.93 & 0.71 \\
\hline 61 & 0.15 & 0.83 & 11 & 15 & 0.81 & 0.76 & 0.86 \\
\hline 71 & 0.01 & 0.93 & 11 & 13 & 0.94 & 0.92 & 0.98 \\
\hline 84 & 0.12 & 0.89 & 11 & 14 & 0.87 & 0.86 & 0.83 \\
\hline
\end{tabular}

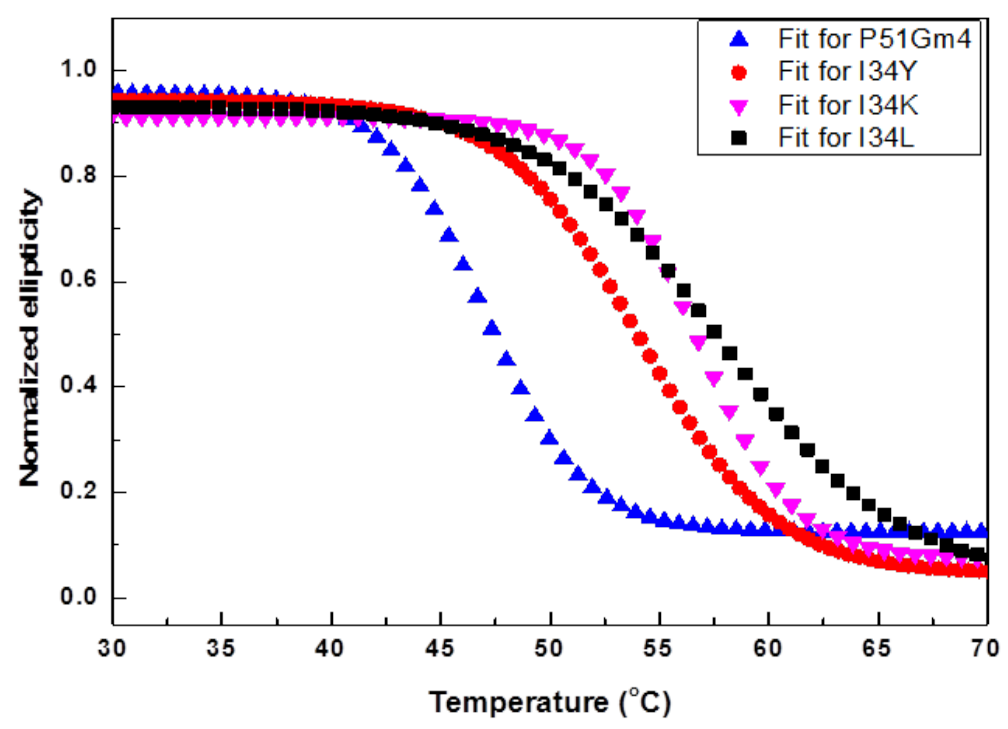

Fig S1 Fits for thermal melts of the CV-N mutants 

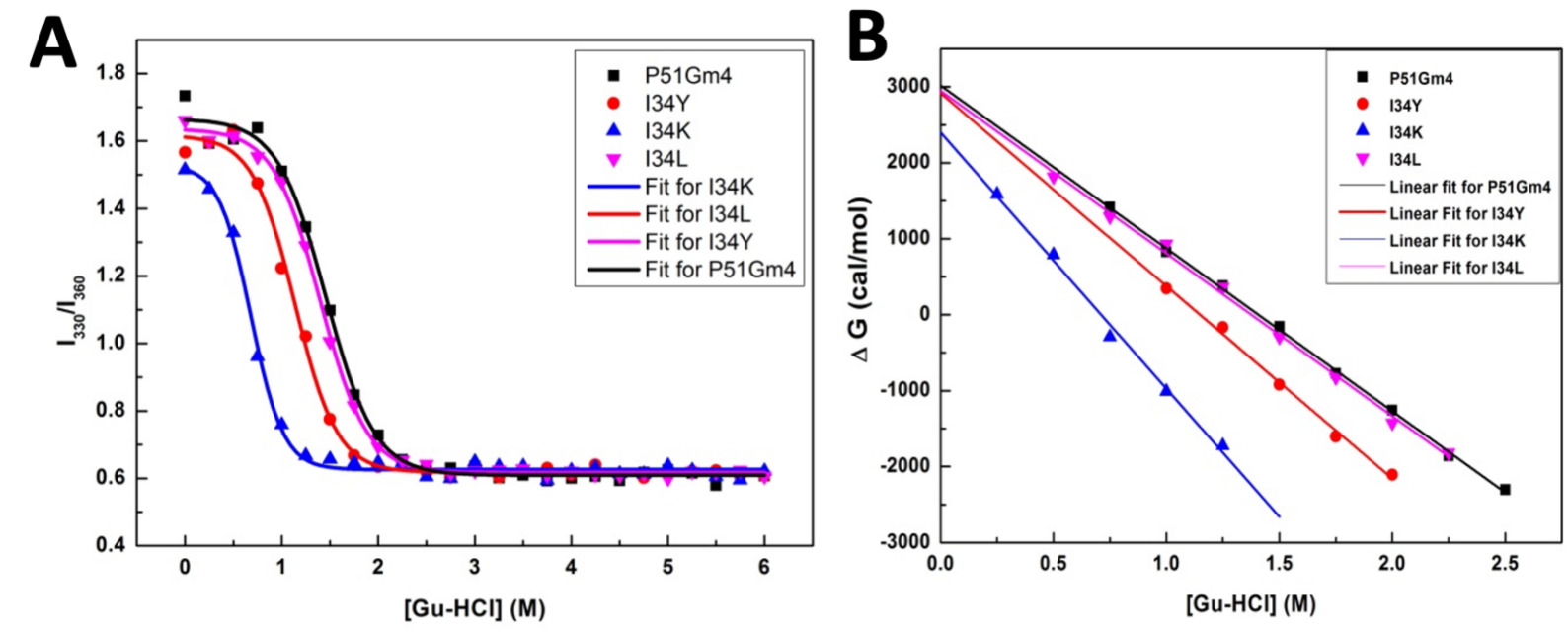

Fig S2 A) Chemical denaturation curve showing $I_{330} / I_{360}$ ratio as a function of $\mathrm{Gu}-\mathrm{HCl}$ concentration, b) $\Delta \mathrm{G}_{\mathrm{H} 2 \mathrm{O}}$ versus $\mathrm{Gu}-\mathrm{HCl}$ concentration plot for $\mathrm{CV}-\mathrm{N}$ mutants.
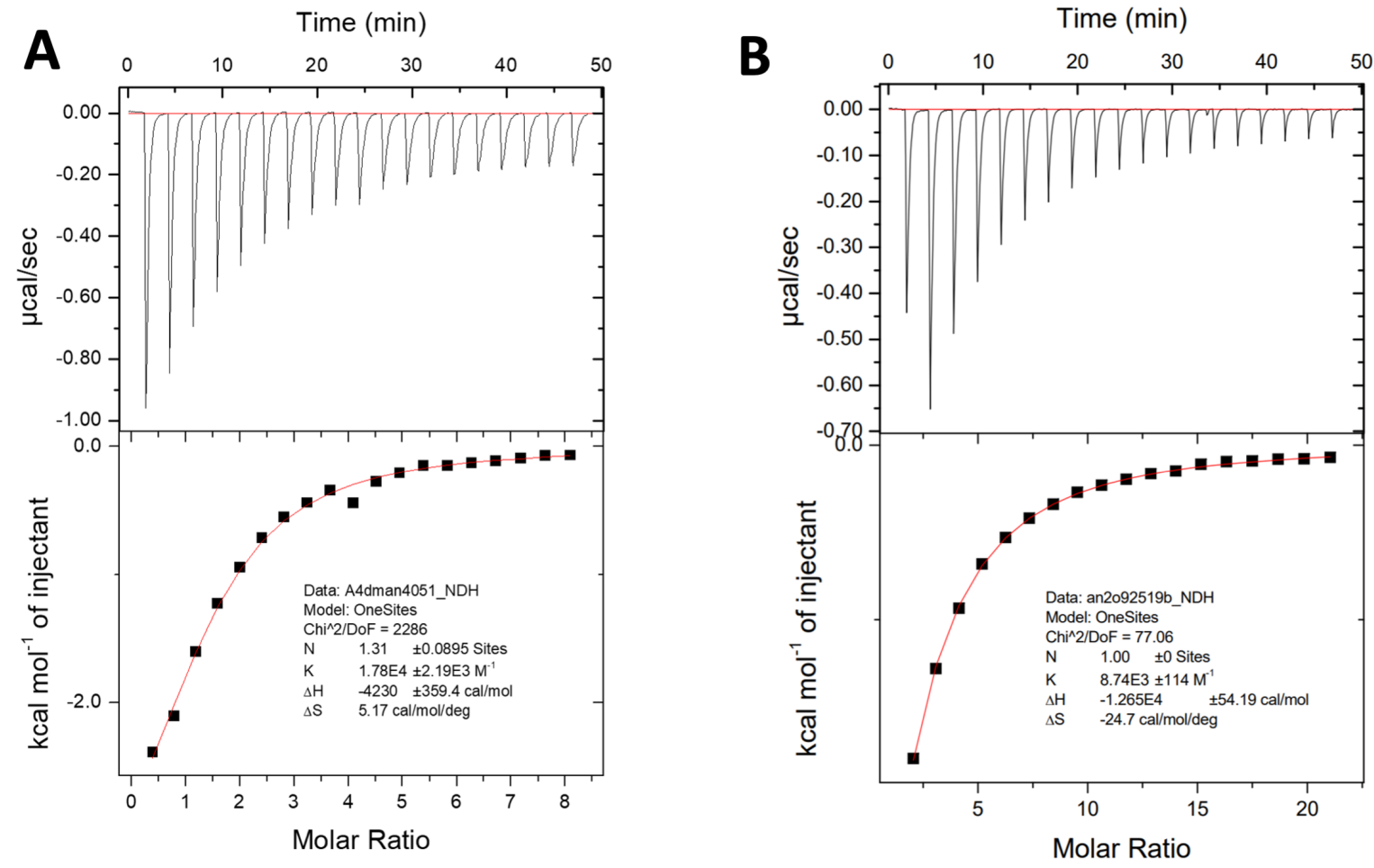

Fig S3 Binding isotherms of CV-N mutants upon titration with dimannose: A) I34Y and

B) P51G-m4 

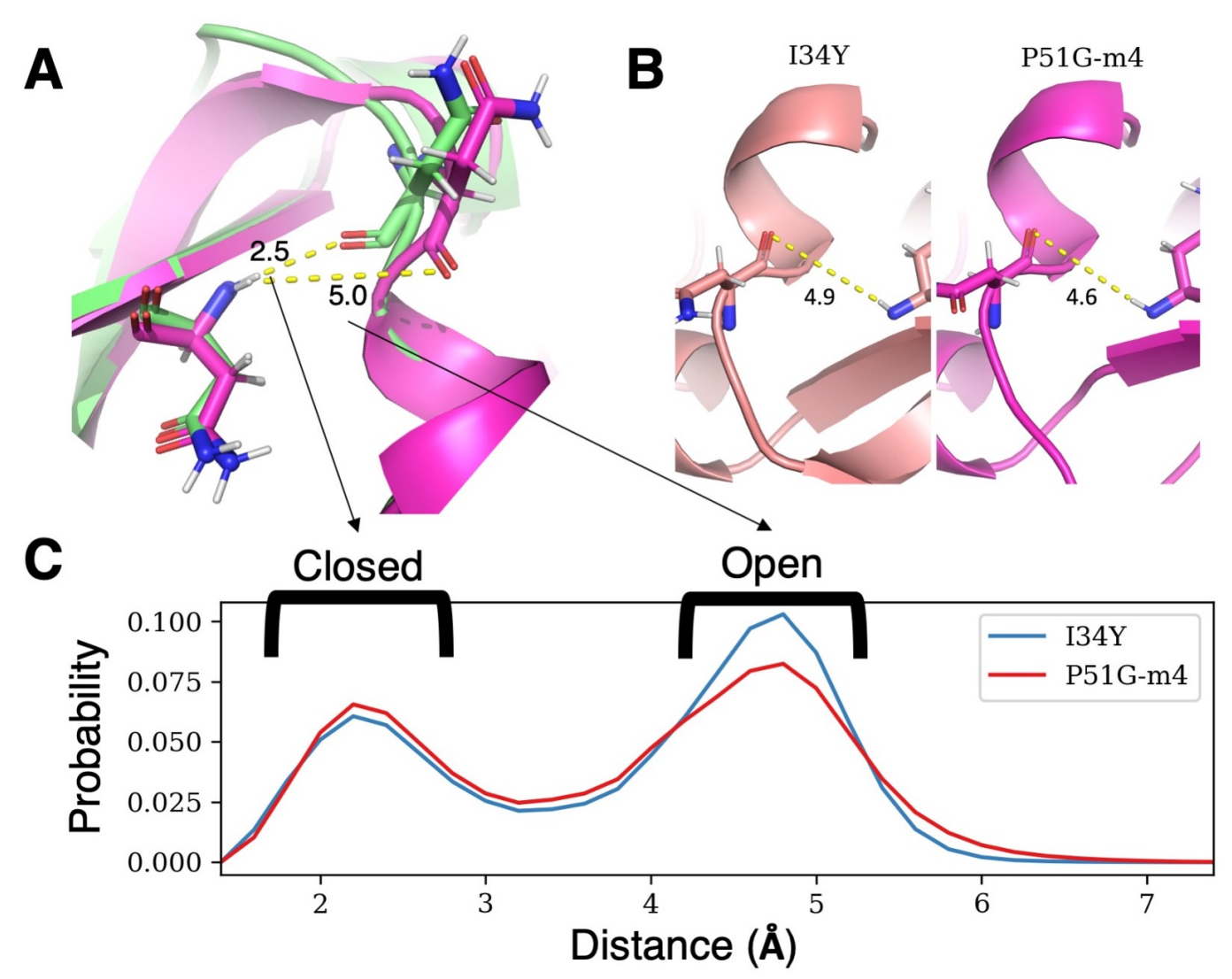

Fig S4 A) Structural difference of open vs closed conformation based on the hydrogen bond distance between residue N42 and N53 B) Hydrogen bond distance between residue N42 and N53 from crystal structures of P51G-m4, and P51G-m4-I34Y C) Frequencies of hydrogen bond distance between residue N42 and N53 from GROMACS production runs showing I34Y variant sampling more open conformation compared to P51G-m4. 


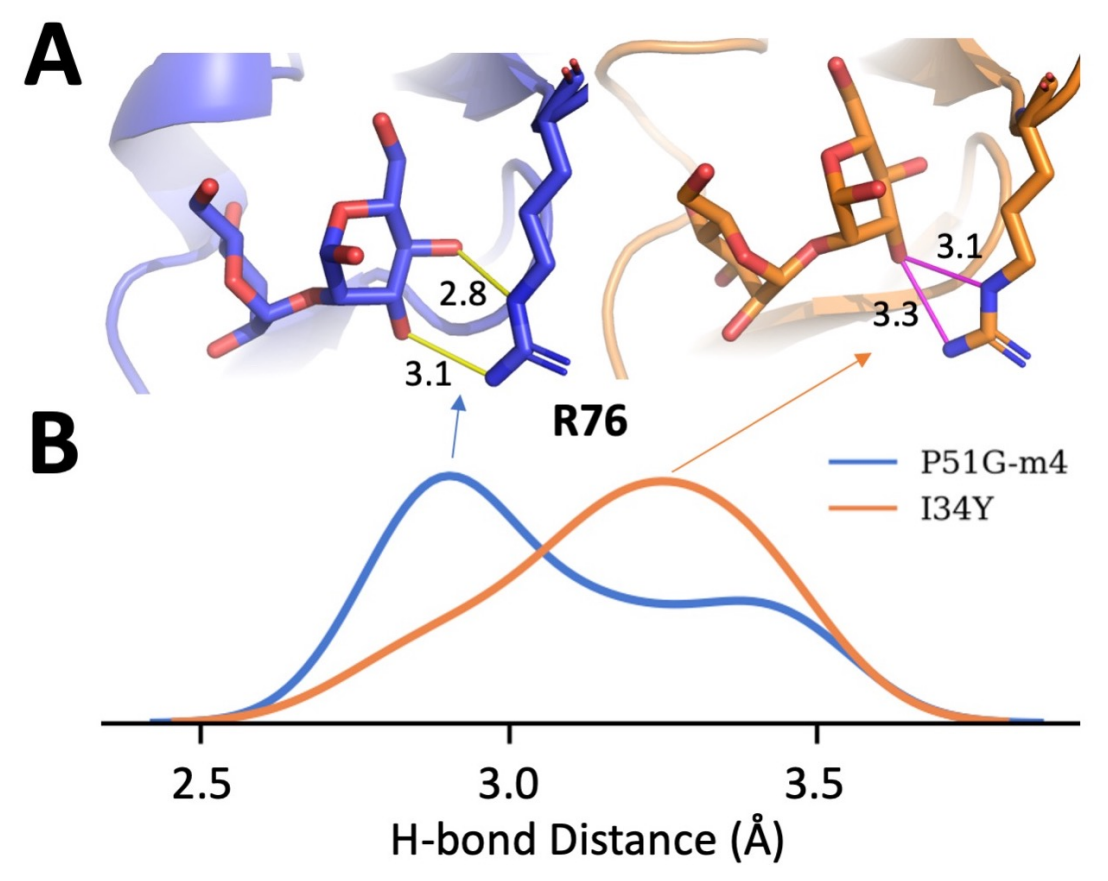

Fig S5 We sampled 2000 different conformations from MD simulations for P51G-m4 CV-N and I34Y mutant and performed dimannose docking to obtained docked poses and then analyzed hydrogen bond patterns A) Hydrogen bonds (representing the peak of the distribution on panel B) and their distances are shown between dimannose and residue R76 for P51G-m4 (blue) and I34Y (orange) B) H-bond distance distribution between dimannose and residue R76. 


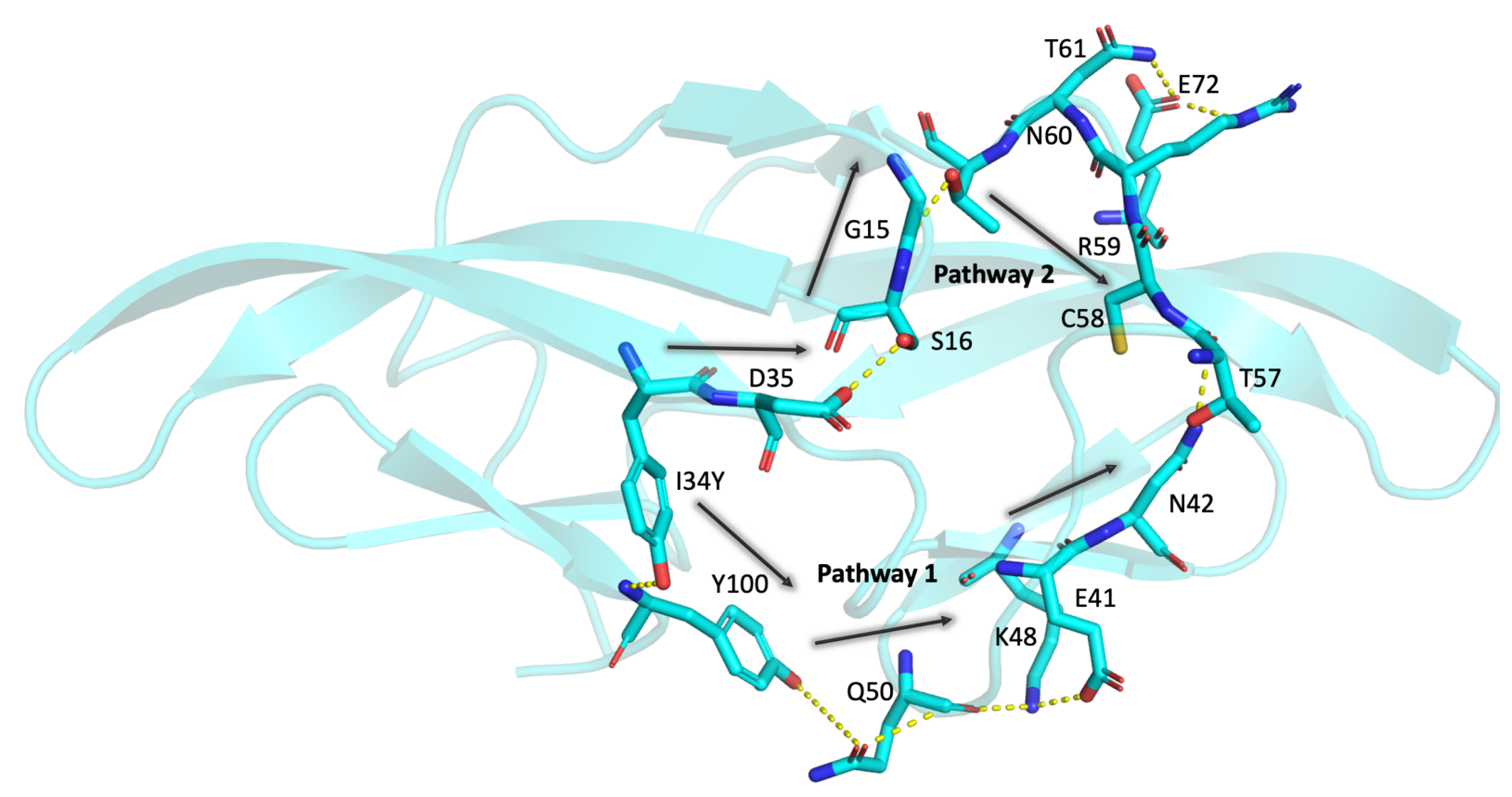

Fig S6 Network of hydrogen bond interactions connecting residue location 34 to T57 is investigated in I34Y variant and P51G-m4 CV-N. Two Hydrogen bond pathways are found connecting residue 34 to 57 . Pathway 1 is unique to $134 \mathrm{Y}$. Pathway 2 is also observed in P51G-m4 CV-N but sampled much more frequently in I34Y variant.

\section{$628 \quad$ Additional files}

630 Supplementary file 1

632 This file contains Supplementary Tables 1 and 2 (referenced in the main text).

634 Supplementary Table 1 contains the complete DFI, DCI, RaptorX, Evcoupling, and MISTIC

635 metric data used in this study. Supplementary Table 2 contains the sequence variations with 
636 binding residues from MSA used in this study to identify possible amino acid variants on

637 location I34. Previous mutational study (Bolia et al., 2014b) on binding residues shown that, N42

638 is highly sequentially conserved as N. Position E41 has two high frequency variants E and G that

639 do not abolish binding. Residue T57 has high frequency variants $\mathrm{S}$ and $\mathrm{T}$, and a rare case $\mathrm{V}$.

640 From the amino acid variation data with binding sites, I34 variants L and Y are selected based on

641 their high frequency and $\mathrm{K}$ as a rare case.

642

\section{References}

645 Abraham MJ, Murtola T, Schulz R, Páll S, Smith JC, Hess B, Lindahl E. 2015. GROMACS:

646 High performance molecular simulations through multi-level parallelism from laptops to

647 supercomputers. SoftwareX 1-2:19-25. doi:10.1016/j.softx.2015.06.001

648 Acar B, Rose J, Aykac Fas B, Ben-Tal N, Lewinson O, Haliloglu T. 2020. Distinct Allosteric

649 Networks Underlie Mechanistic Speciation of ABC Transporters. Structure 28:651-

$650 \quad$ 663.e5. doi:10.1016/j.str.2020.03.014

651 Anishchenko I, Ovchinnikov S, Kamisetty H, Baker D. 2017. Origins of coevolution between residues distant in protein 3D structures. PNAS 114:9122-9127. doi:10.1073/pnas.1702664114

654 Atilgan AR, Durell SR, Jernigan RL, Demirel MC, Keskin O, Bahar I. 2001. Anisotropy of Fluctuation Dynamics of Proteins with an Elastic Network Model. Biophysical Journal 80:505-515. doi:10.1016/S0006-3495(01)76033-X 
Atilgan C, Atilgan AR. 2009. Perturbation-Response Scanning Reveals Ligand Entry-Exit Mechanisms of Ferric Binding Protein. PLoS Comput Biol 5. doi:10.1371/journal.pcbi.1000544

Atilgan C, Gerek ZN, Ozkan SB, Atilgan AR. 2010. Manipulation of Conformational Change in Proteins by Single-Residue Perturbations. Biophysical Journal 99:933-943. doi:10.1016/j.bpj.2010.05.020

Balzarini J. 2007. Targeting the glycans of glycoproteins: a novel paradigm for antiviral therapy. Nature Reviews Microbiology 5:583-597. doi:10.1038/nrmicro1707

Barrientos LG, Matei E, Lasala F, Delgado R, Gronenborn AM. 2006. Dissecting carbohydrateCyanovirin-N binding by structure-guided mutagenesis: functional implications for viral entry inhibition. Protein Eng Des Sel 19:525-535. doi:10.1093/protein/gz1040

Barrientos LG, O’Keefe BR, Bray M, Sanchez A, Gronenborn AM, Boyd MR. 2003. Cyanovirin-N binds to the viral surface glycoprotein, GP1,2 and inhibits infectivity of Ebola virus. Antiviral Research 58:47-56. doi:10.1016/S0166-3542(02)00183-3

674 Bolia A, Gerek ZN, Ozkan SB. 2014a. BP-Dock: A Flexible Docking Scheme for Exploring Protein-Ligand Interactions Based on Unbound Structures. J Chem Inf Model 54:913925. doi:10.1021/ci4004927

Bolia A, Ozkan SB. 2016. Adaptive BP-Dock: An Induced Fit Docking Approach for Full Receptor Flexibility. J Chem Inf Model 56:734-746. doi:10.1021/acs.jcim.5b00587 
Bolia A, Woodrum BW, Cereda A, Ruben MA, Wang X, Ozkan SB, Ghirlanda G. 2014b. A Flexible Docking Scheme Efficiently Captures the Energetics of Glycan-Cyanovirin Binding. Biophysical Journal 106:1142-1151. doi:10.1016/j.bpj.2014.01.040

Bolmstedt AJ, O’Keefe BR, Shenoy SR, McMahon JB, Boyd MR. 2001. Cyanovirin-N Defines a New Class of Antiviral Agent Targeting N-Linked, High-Mannose Glycans in an Oligosaccharide-Specific Manner. Mol Pharmacol 59:949-954.

Botos I, Wlodawer A. 2005. Proteins that bind high-mannose sugars of the HIV envelope. Progress in Biophysics and Molecular Biology, Structure-guided design of AIDs Antivirals 88:233-282. doi:10.1016/j.pbiomolbio.2004.05.001

Botos I, Wlodawer A. 2003. Cyanovirin-N: a sugar-binding antiviral protein with a new twist. CMLS, Cell Mol Life Sci 60:277-287. doi:10.1007/s000180300023

Butler BM, Gerek ZN, Kumar S, Ozkan SB. 2015. Conformational dynamics of nonsynonymous variants at protein interfaces reveals disease association. Proteins: Structure, Function, and Bioinformatics 83:428-435. doi:10.1002/prot.24748

694 Campbell E, Kaltenbach M, Correy GJ, Carr PD, Porebski BT, Livingstone EK, Afriat-Jurnou L, Buckle AM, Weik M, Hollfelder F, Tokuriki N, Jackson CJ. 2016. The role of protein dynamics in the evolution of new enzyme function. Nature Chemical Biology 12:944950. doi:10.1038/nchembio. 2175 
701 Campitelli P, Guo J, Zhou H-X, Ozkan SB. 2018. Hinge-Shift Mechanism Modulates Allosteric

702 Regulations in Human Pin1. J Phys Chem B 122:5623-5629.

703 doi:10.1021/acs.jpcb.7b11971

704 Campitelli P, Modi T, Kumar S, Ozkan SB. 2020a. The Role of Conformational Dynamics and

705 Allostery in Modulating Protein Evolution. Annual Review of Biophysics 49:267-288. doi:10.1146/annurev-biophys-052118-115517

707 Campitelli P, Ozkan SB, Swint-Kruse L. 2020b. Asymmetry in Dynamic Allosteric Residue Coupling (DARC) Interactions Captures Evolutionary Landscape. Biophysical Journal

Chang LC, Bewley CA. 2002. Potent Inhibition of HIV-1 Fusion by Cyanovirin-N Requires doi:10.1016/S0022-2836(02)00045-1

714 Davis IW, Baker D. 2009. RosettaLigand Docking with Full Ligand and Receptor Flexibility. Journal of Molecular Biology 385:381-392. doi:10.1016/j.jmb.2008.11.010

716 Davis IW, Raha K, Head MS, Baker D. 2009. Blind docking of pharmaceutically relevant compounds using RosettaLigand. Protein Science 18:1998-2002. doi:10.1002/pro.192

718 de Juan D, Pazos F, Valencia A. 2013. Emerging methods in protein co-evolution. Nature Reviews Genetics 14:249-261. doi:10.1038/nrg3414 
Dunn SD, Wahl LM, Gloor GB. 2008. Mutual information without the influence of phylogeny or entropy dramatically improves residue contact prediction. Bioinformatics 24:333-340. doi:10.1093/bioinformatics/btm604

725

726

Fromme R, Katiliene Z, Giomarelli B, Bogani F, Mc Mahon J, Mori T, Fromme P, Ghirlanda G. binding to the antiviral protein cyanovirin revealed from the crystal structure at $1.35 \AA$ resolution. Protein Science 17:939-944. doi:10.1110/ps.083472808 2007. A Monovalent Mutant of Cyanovirin-N Provides Insight into the Role of Multiple Interactions with gp120 for Antiviral Activity,. Biochemistry 46:9199-9207. doi:10.1021/bi700666m

Fujimoto YK, Green DF. 2012. Carbohydrate Recognition by the Antiviral Lectin Cyanovirin-N. J Am Chem Soc 134:19639-19651. doi:10.1021/ja305755b

Gerek ZN, Kumar S, Ozkan SB. 2013. Structural dynamics flexibility informs function and evolution at a proteome scale. Evolutionary Applications 6:423-433. doi:10.1111/eva.12052

Gerek ZN, Ozkan SB. 2011. Change in Allosteric Network Affects Binding Affinities of PDZ Domains: Analysis through Perturbation Response Scanning. PLOS Computational Biology 7:e1002154. doi:10.1371/journal.pcbi.1002154

Gouveia-Oliveira R, Pedersen AG. 2007. Finding coevolving amino acid residues using row and column weighting of mutual information and multi-dimensional amino acid representation. Algorithms for Molecular Biology 2:12. doi:10.1186/1748-7188-2-12

Gunasekaran K, Ma B, Nussinov R. 2004. Is allostery an intrinsic property of all dynamic proteins? Proteins: Structure, Function, and Bioinformatics 57:433-443. 
745 Hopf TA, Green AG, Schubert B, Mersmann S, Schärfe CPI, Ingraham JB, Toth-Petroczy A, Brock K, Riesselman AJ, Palmedo P, Kang C, Sheridan R, Draizen EJ, Dallago C, Sander C, Marks DS. 2019. The EVcouplings Python framework for coevolutionary

Hopf TA, Schärfe CPI, Rodrigues JPGLM, Green AG, Kohlbacher O, Sander C, Bonvin AMJJ, Marks DS. 2018. Sequence co-evolution gives 3D contacts and structures of protein

Ikeguchi M, Ueno J, Sato M, Kidera A. 2005. Protein Structural Change Upon Ligand Binding:

Jana B, Morcos F, Onuchic JN. 2014. From structure to function: the convergence of structure based models and co-evolutionary information. Phys Chem Chem Phys 16:6496-6507. doi:10.1039/C3CP55275F

Jones DT, Buchan DWA, Cozzetto D, Pontil M. 2012. PSICOV: precise structural contact prediction using sparse inverse covariance estimation on large multiple sequence alignments. Bioinformatics 28:184-190. doi:10.1093/bioinformatics/btr638

Kamisetty H, Ovchinnikov S, Baker D. 2013. Assessing the utility of coevolution-based residueresidue contact predictions in a sequence- and structure-rich era. PNAS 110:15674-

763 Keeffe JR, Gnanapragasam PNP, Gillespie SK, Yong J, Bjorkman PJ, Mayo SL. 2011. Designed oligomers of cyanovirin-N show enhanced HIV neutralization. PNAS 108:14079-14084. doi:10.1073/pnas.1108777108 
Kelley BS, Chang LC, Bewley CA. 2002. Engineering an Obligate Domain-Swapped Dimer of doi:10.1021/ja025537m

Koharudin LMI, Furey W, Gronenborn AM. 2009. A designed chimeric cyanovirin-N homolog lectin: Structure and molecular basis of sucrose binding. Proteins: Structure, Function,

Koharudin LMI, Gronenborn AM. 2013. Sweet entanglements-protein: Glycan interactions in two HIV-inactivating lectin families. Biopolymers 99:196-202. doi:10.1002/bip.22106

Koharudin LMI, Viscomi AR, Jee J-G, Ottonello S, Gronenborn AM. 2008. The Evolutionarily Conserved Family of Cyanovirin-N Homologs: Structures and Carbohydrate Specificity.

Kryshtafovych A, Schwede T, Topf M, Fidelis K, Moult J. 2019. Critical assessment of methods Bioinformatics 87:1011-1020. doi:10.1002/prot.25823

Kumar A, Butler BM, Kumar S, Ozkan SB. 2015a. Integration of structural dynamics and molecular evolution via protein interaction networks: a new era in genomic medicine. Current Opinion in Structural Biology, Catalysis and regulation • Protein-protein interactions 35:135-142. doi:10.1016/j.sbi.2015.11.002

Kuriyan J, Eisenberg D. 2007. The origin of protein interactions and allostery in colocalization. 
Larrimore KE, Kazan IC, Kannan L, Kendle RP, Jamal T, Barcus M, Bolia A, Brimijoin S, Zhan C-G, Ozkan SB. 2017. Plant-expressed cocaine hydrolase variants of butyrylcholinesterase exhibit altered allosteric effects of cholinesterase activity and

Li Z, Bolia A, Maxwell JD, Bobkov AA, Ghirlanda G, Ozkan SB, Margulis CJ. 2015. A Rigid Hinge Region Is Necessary for High-Affinity Binding of Dimannose to Cyanovirin and Natl Acad Sci U S A 114:7480-7482. doi:10.1073/pnas.1709071114

Lu H-M, Liang J. 2009. Perturbation-based Markovian transmission model for probing allosteric dynamics of large macromolecular assembling: a study of GroEL-GroES. PLoS Comput Associated Constructs. Biochemistry 54:6951-6960. doi:10.1021/acs.biochem.5b00635

Liu J, Nussinov R. 2017. Energetic redistribution in allostery to execute protein function. Proc

Marks DS, Hopf TA, Sander C. 2012. Protein structure prediction from sequence variation. Nature Biotechnology 30:1072-1080. doi:10.1038/nbt.2419

Matei E, Basu R, Furey W, Shi J, Calnan C, Aiken C, Gronenborn AM. 2016. Structure and Glycan Binding of a New Cyanovirin-N Homolog. J Biol Chem 291:18967-18976. Biol 5:e1000526. doi:10.1371/journal.pcbi.1000526

Ma J, Wang S, Wang Z, Xu J. 2015. Protein contact prediction by integrating joint evolutionary coupling analysis and supervised learning. Bioinformatics 31:3506-3513. 1194. doi:10.1016/j.str.2008.05.011 
812 Matei E, Louis JM, Jee J, Gronenborn AM. 2011. NMR solution structure of a cyanovirin homolog from wheat head blight fungus. Proteins: Structure, Function, and Bioinformatics 79:1538-1549. doi:10.1002/prot.22981

815 Meiler J, Baker D. 2006. ROSETTALIGAND: Protein-small molecule docking with full sidechain flexibility. Proteins: Structure, Function, and Bioinformatics 65:538-548.

Modi T, Campitelli P, Kazan IC, Ozkan SB. 2021. Protein folding stability and binding interactions through the lens of evolution: a dynamical perspective. Current Opinion in

Modi T, Ozkan SB. 2018. Mutations Utilize Dynamic Allostery to Confer Resistance in TEM-1

Morcos F. 2020. Protein conformations à la carte, a step further in de novo protein design. PNAS

$$
\text { 117:8674-8676. doi:10.1073/pnas.2004188117 }
$$

Morcos F, Jana B, Hwa T, Onuchic JN. 2013. Coevolutionary signals across protein lineages help capture multiple protein conformations. PNAS 110:20533-20538. contacts across many protein families. PNAS 108:E1293-E1301.

832 Morcos F, Schafer NP, Cheng RR, Onuchic JN, Wolynes PG. 2014. Coevolutionary information, protein folding landscapes, and the thermodynamics of natural selection. PNAS 111:12408-12413. doi:10.1073/pnas.1413575111 
Mori T, Boyd MR. 2001. Cyanovirin-N, a Potent Human Immunodeficiency Virus-Inactivating Protein, Blocks both CD4-Dependent and CD4-Independent Binding of Soluble gp120 (sgp120) to Target Cells, Inhibits sCD4-Induced Binding of sgp120 to Cell-Associated

O'Keefe BR, Smee DF, Turpin JA, Saucedo CJ, Gustafson KR, Mori T, Blakeslee D, Buckheit Viral Hemagglutinin. Antimicrobial Agents and Chemotherapy 47:2518-2525.

Ose N, Butler BM, Kumar A, Ozkan SB, Kumar S. 2020. Dynamic Allosteric Residue Coupling Reveals Disease Mechanism for Gaucher Disease and NSNVS Across the Proteome.

847 Otten R, Liu L, Kenner LR, Clarkson MW, Mavor D, Tawfik DS, Kern D, Fraser JS. 2018. Rescue of conformational dynamics in enzyme catalysis by directed evolution. Nature

850 Patsalo V, Raleigh DP, Green DF. 2011. Rational and Computational Design of Stabilized Variants of Cyanovirin-N That Retain Affinity and Specificity for Glycan Ligands.

853 Percudani R, Montanini B, Ottonello S. 2005. The anti-HIV cyanovirin-N domain is evolutionarily conserved and occurs as a protein module in eukaryotes. Proteins: Structure, Function, and Bioinformatics 60:670-678. doi:10.1002/prot.20543 
Qi X, Yang Y, Su Y, Wang T. 2009. Molecular Cloning and Sequence Analysis of Cyanovirin-N 99.2.78

859 Ramadugu SK, Li Z, Kashyap HK, Margulis CJ. 2014. The Role of Glu41 in the Binding of Dimannose to P51G-m4-CVN. Biochemistry 53:1477-1484. doi:10.1021/bi4014159

Risso VA, Sanchez-Ruiz JM, Ozkan SB. 2018. Biotechnological and protein-engineering implications of ancestral protein resurrection. Current Opinion in Structural Biology, Engineering and design: New applications • Membranes 51:106-115. doi:10.1016/j.sbi.2018.02.007

Romero PA, Arnold FH. 2009. Exploring protein fitness landscapes by directed evolution. Nature Reviews Molecular Cell Biology 10:866-876. doi:10.1038/nrm2805

Salinas VH, Ranganathan R. 2018. Coevolution-based inference of amino acid interactions underlying protein function. eLife 7:e34300. doi:10.7554/eLife.34300

869 Simonetti FL, Teppa E, Chernomoretz A, Nielsen M, Marino Buslje C. 2013. MISTIC: mutual information server to infer coevolution. Nucleic Acids Res 41:W8-W14.

Spoel DVD, Lindahl E, Hess B, Groenhof G, Mark AE, Berendsen HJC. 2005. GROMACS: Fast, flexible, and free. Journal of Computational Chemistry 26:1701-1718.

875 Tokuriki N, Jackson CJ, Afriat-Jurnou L, Wyganowski KT, Tang R, Tawfik DS. 2012. 
Tripathi S, Waxham MN, Cheung MS, Liu Y. 2015. Lessons in Protein Design from Combined Evolution and Conformational Dynamics. Scientific Reports 5:14259. doi:10.1038/srep14259

Vorontsov II, Miyashita O. 2009. Solution and Crystal Molecular Dynamics Simulation Study of m4-Cyanovirin-N Mutants Complexed with Di-Mannose. Biophysical Journal 97:25322540. doi:10.1016/j.bpj.2009.08.011

Wagner JR, Sørensen J, Hensley N, Wong C, Zhu C, Perison T, Amaro RE. 2017. POVME 3.0: Software for Mapping Binding Pocket Flexibility. J Chem Theory Comput 13:4584-

Wang R, Lai L, Wang S. 2002. Further development and validation of empirical scoring 26. doi:10.1023/A:1016357811882

Wang S, Li W, Zhang R, Liu S, Xu J. 2016. CoinFold: a web server for protein contact prediction and contact-assisted protein folding. Nucleic Acids Res 44:W361-W366.

Wang S, Sun S, Li Z, Zhang R, Xu J. 2017. Accurate De Novo Prediction of Protein Contact doi:10.1093/nar/gkw307

Wodak SJ, Paci E, Dokholyan NV, Berezovsky IN, Horovitz A, Li J, Hilser VJ, Bahar I, 
D, McLeish T. 2019. Allostery in Its Many Disguises: From Theory to Applications.

902 Structure 27:566-578. doi:10.1016/j.str.2019.01.003

903 Woodrum BW, Maxwell JD, Bolia A, Ozkan SB, Ghirlanda G. 2013. The antiviral lectin

904 cyanovirin-N: probing multivalency and glycan recognition through experimental and

905 computational approaches. Biochem Soc Trans 41:1170-1176.

906 doi:10.1042/BST20130154

907 Yuan L, Kurek I, English J, Keenan R. 2005. Laboratory-Directed Protein Evolution. Microbiol Mol Biol Rev 69:373-392. doi:10.1128/MMBR.69.3.373-392.2005

909 Zhang X, Gureasko J, Shen K, Cole PA, Kuriyan J. 2006. An allosteric mechanism for activation

$910 \quad$ of the kinase domain of epidermal growth factor receptor. Cell 125:1137-1149.

$911 \quad$ doi:10.1016/j.cell.2006.05.013

912

913

914

915 